\title{
Profinite invariants of arithmetic groups
}

\author{
Holger Kammeyer ${ }^{(\mathbb{D} 1}{ }$, Steffen Kionke ${ }^{\left(\mathbb{D}_{2}\right.}$, Jean Raimbault ${ }^{\left(\mathbb{D}_{3}\right.}$ and Roman Sauer ${ }^{(\mathbb{D}} 1$ \\ ${ }^{1}$ Institute for Algebra and Geometry, Karlsruhe Institute of Technology, 76131 Karlsruhe, Germany; \\ E-mail: holger.kammeyer@kit.edu, roman.sauer@kit.edu. \\ ${ }^{2}$ Faculty of Mathematics and Computer Science, FernUniversität in Hagen, 58097 Hagen, Germany; \\ E-mail: steffen.kionke@fernuni-hagen.de. \\ ${ }^{3}$ Institut de Mathématiques de Toulouse; UMR5219 Université de Toulouse; CNRS UPS IMT, F-31062 Toulouse Cedex 9, \\ France; E-mail: Jean.Raimbault@ math.univ-toulouse.fr.
}

Received: 19 March 2020; Revised: 19 May 2020; Accepted: 4 June 2020

Keywords and phrases: profinite rigidity, arithmetic groups, 12-invariants

2020 Mathematics Subject Classification: Primary-20E18; Secondary-11F75

\begin{abstract}
We prove that the sign of the Euler characteristic of arithmetic groups with the congruence subgroup property is determined by the profinite completion. In contrast, we construct examples showing that this is not true for the Euler characteristic itself and that the sign of the Euler characteristic is not profinite among general residually finite groups of type $F$. Our methods imply similar results for $\ell^{2}$-torsion as well as a strong profiniteness statement for Novikov-Shubin invariants.
\end{abstract}

\section{Contents}

1 Introduction 1

1.1 Main results . . . . . . . . . . . . . . . . . . . . 2

1.2 Extension to other invariants . . . . . . . . . . . . . . . 3

1.3 Toward $S$-arithmetic groups and weakening CSP . . . . . . . . . . . . . 4

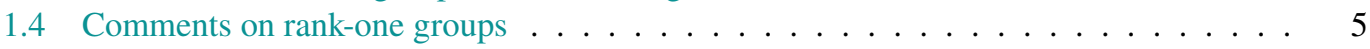

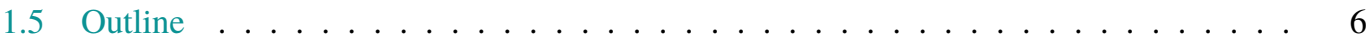

2 Profinite invariance of fundamental rank and dimension mod $4 \quad 6$

3 Conclusion of main results $\quad 11$

4 The Euler characteristic of arithmetic spin groups $\quad 13$

\section{Introduction}

A finitely generated, residually finite group $\Gamma$ is called profinitely rigid if any other such group $\Lambda$ with the same set of finite quotients as $\Gamma$ is isomorphic to $\Gamma$; this can be expressed in terms of profinite completions: if $\widehat{\Lambda} \cong \widehat{\Gamma}$, then $\Lambda \cong \Gamma$ (see [19]). While all finitely generated abelian groups have this property, there are already virtually cyclic groups that are not profinitely rigid [5]. In general, profinite rigidity is extremely difficult to characterize. Recent work of Bridson, McReynolds, Reid, and Spitler [12] shows that profinite rigidity holds for certain Kleinian groups, including the Weeks manifold group. On the other hand, we note that profinite rigidity of free groups, surface groups, or $\mathrm{SL}_{n}(\mathbb{Z})$ is still open. 


\subsection{Main results}

Two related questions seem more accessible: (i) to establish profinite rigidity among a certain class of groups and (ii) to find profinite invariants. A group invariant is profinite if it takes the same value on finitely generated, residually finite groups whose profinite completions are isomorphic. In this paper, we study a combination of both: we establish profinite invariance of the sign of the Euler characteristic within a most relevant subclass of finitely generated, residually finite groups: arithmetic groups with the congruence subgroup property. In particular, this (conjecturally) includes all irreducible lattices in higher-rank semisimple Lie groups.

Theorem 1.1. Let $\underline{G}_{1}$ and $\underline{G}_{2}$ be linear algebraic groups defined over number fields $k_{1}$ and $k_{2}$, and let $\Gamma_{1} \leq \underline{G}_{1}\left(k_{1}\right)$ and $\Gamma_{2} \leq \underline{G}_{2}\left(k_{2}\right)$ be arithmetic subgroups. Suppose that $\underline{G}_{1}$ and $\underline{G}_{2}$ have a finite congruence kernel and that $\Gamma_{1}$ is profinitely commensurable with $\Gamma_{2}$. Then $\operatorname{sign} \chi\left(\Gamma_{1}\right)=\operatorname{sign} \chi\left(\Gamma_{2}\right)$.

Let us explain the meaning of the terms in this statement. Two groups $\Gamma_{1}$ and $\Gamma_{2}$ are called profinitely commensurable if the profinite completions $\widehat{\Gamma_{1}}$ and $\widehat{\Gamma_{2}}$ have isomorphic open subgroups. Equivalently, $\Gamma_{1}$ and $\Gamma_{2}$ have finite index subgroups with isomorphic profinite completions. The function $\operatorname{sign}(x)$ takes the values $-1,0,1$ if $x<0, x=0, x>0$. A subgroup of $\underline{G}(k)$ is arithmetic if it is commensurable to $\underline{G}\left(\mathcal{O}_{k}\right):=\underline{G}(k) \cap \mathrm{GL}_{n}\left(\mathcal{O}_{k}\right)$ for some $k$-rational embedding $\underline{G} \rightarrow \mathrm{GL}_{n}$, where $\mathcal{O}_{k}$ is the ring of algebraic integers in $k$. Any nonzero ideal $\mathfrak{a} \subset \mathcal{O}_{k}$ defines a finite index normal subgroup of $\underline{G}\left(\mathcal{O}_{k}\right)$ as the kernel of the homomorphism $\underline{G}\left(\mathcal{O}_{k}\right) \rightarrow \underline{G}\left(\mathcal{O}_{k} / \mathfrak{a}\right)$ defined by reduction of coefficients. These are called principal congruence subgroups. They define a unit neighborhood base in $\underline{G}(k)$ for the socalled congruence topology. The arithmetic topology of $\underline{G}(k)$, defined by declaring all finite index subgroups of $\underline{G}\left(\mathcal{O}_{k}\right)$ as a unit neighborhood base, is thus a priori finer than the congruence topology. It is straightforward to see that neither topology depends on the chosen embedding $G \rightarrow \mathrm{GL}_{n}$. Hence we obtain a canonical continuous homomorphism $\overline{G(k)} \rightarrow \overline{G(k)}$ from the arithmetic completion to the congruence completion, where each completion is formed with respect to the canonical uniform structure of topological groups. The kernel is called the congruence kernel of $\underline{G}$. Serre conjectured that for simple groups $\underline{G}$, the congruence kernel is finite (' $\underline{G}$ has the congruence subgroup property') whenever the real Lie group $\underline{G}\left(k \otimes_{\mathbb{Q}} \mathbb{R}\right)$ has real rank at least 2; we refer to [50] for a survey on the status of this conjecture. A purely group theoretic characterization of the congruence subgroup property can be given in terms of subgroup growth, and it is also closely related to the notion of bounded generation [41].

We note that it is known that profinite rigidity does not hold for all higher-rank arithmetic lattices, even among themselves (as follows from [2]). However, the profinite isomorphism class of arithmetic groups for which the congruence subgroup property holds is easier to understand than that of general lattices; for example, M. Aka proves in [2] that it is always finite within the class of arithmetic groups. To prove the theorem above, we push Aka's arguments further.

It is possible to calculate the Euler characteristic of arithmetic groups using Harder's Gauß-Bonnet theorem [25]. We apply this method to obtain the following example, which shows that Theorem 1.1 does not extend to the Euler characteristic itself.

Theorem 1.2. For positive integers $m$ and $n$, let $\Gamma_{m, n}$ be the level-four principal congruence subgroup of $\operatorname{Spin}(m, n)(\mathbb{Z})$. Then $\widehat{\Gamma_{8,2}} \cong \widehat{\Gamma_{4,6}}$, but

$$
\chi\left(\Gamma_{8,2}\right)=2^{89} \cdot 5^{2} \cdot 17 \quad \text { whereas } \chi\left(\Gamma_{4,6}\right)=2^{90} \cdot 5^{2} \cdot 17
$$

The spinor groups $\operatorname{Spin}(m, n)(\mathbb{Z})$ arise from the $(m+n)$-ary integral diagonal quadratic form with $m$ coefficients ' +1 ' and $n$ coefficients ' -1 '. Precise definitions are given in Section 4 . The existence of the above examples implies that one cannot broaden the conclusion of Theorem 1.1 from arithmetic to residually finite groups that admit a finite classifying space. The latter is referred to as being of type $(F)$. 
Corollary 1.3. There are three residually finite groups $\Gamma_{1}, \Gamma_{2}$, and $\Gamma_{3}$ of type $(F)$ that have isomorphic profinite completions such that

$$
\chi\left(\Gamma_{1}\right)<0, \quad \chi\left(\Gamma_{2}\right)=0, \quad \chi\left(\Gamma_{3}\right)>0 .
$$

Setting $c=2^{89} \cdot 5^{2} \cdot 17$, the above groups can simply be taken as

$$
\begin{aligned}
& \Gamma_{1}=\left(\Gamma_{8,2} \times \Gamma_{8,2}\right) * F_{2 c^{2}}, \\
& \Gamma_{2}=\left(\Gamma_{8,2} \times \Gamma_{4,6}\right) * F_{2 c^{2}}, \\
& \Gamma_{3}=\left(\Gamma_{4,6} \times \Gamma_{4,6}\right) * F_{2 c^{2}},
\end{aligned}
$$

where $F_{2 c^{2}}$ is the free group on $2 c^{2}$ letters. Since the profinite completion functor preserves products and coproducts, the three groups are profinitely isomorphic. They are still residually finite and of type $(F)$. Additivity and multiplicativity of the Euler characteristic gives

$$
\begin{aligned}
& \chi\left(\Gamma_{1}\right)=c^{2}+\left(1-2 c^{2}\right)-1=-c^{2}<0, \\
& \chi\left(\Gamma_{2}\right)=2 c^{2}+\left(1-2 c^{2}\right)-1=0, \\
& \chi\left(\Gamma_{3}\right)=4 c^{2}+\left(1-2 c^{2}\right)-1=2 c^{2}>0 .
\end{aligned}
$$

The Euler characteristic equals the alternating sum of the $\ell^{2}$-Betti numbers [42, 31]. For arithmetic groups, $\ell^{2}$-Betti numbers are known to be nonzero in at most one degree. Such a nonzero $\ell^{2}$-Betti number occurs if and only if the group is semisimple and the fundamental rank is zero. In that case, the degree with nonvanishing $\ell^{2}$-Betti number is given by half the dimension of the associated symmetric space $X$ so that we have $\operatorname{sign} \chi(\Gamma)=(-1)^{\operatorname{dim} X / 2}$. This dimension, however, can change when passing to a profinitely commensurable arithmetic group. So $\ell^{2}$-Betti numbers themselves are not profinite. Among $S$-arithmetic groups, no higher $\ell^{2}$-Betti number is profinite [32], in contrast to the first $\ell^{2}$-Betti number that is profinite among all finitely presented residually finite groups [11, Corollary 3.3]. Thus, in the semisimple case, the proof of Theorem 1.1 splits into two parts: showing that the fundamental rank is profinite, so that the vanishing Euler characteristic is profinite, and showing that the profinite completion determines the dimension of the symmetric space mod 4.

\subsection{Extension to other invariants}

Whenever an arithmetic group $\Gamma$ has vanishing Euler characteristic, a secondary invariant called $\ell^{2}$. torsion and denoted by $\rho^{(2)}(\Gamma)$ is defined; see [42, Chapter 3] and [31, Chapter 5] for an introduction. In many ways, $\rho^{(2)}(\Gamma)$ behaves like an 'odd-dimensional cousin' of $\chi(\Gamma)$. Also, the profinite behavior of $\rho^{(2)}(\Gamma)$ is parallel to $\chi(\Gamma)$.

Theorem 1.4. In addition to the assumptions in Theorem 1.1, suppose that $\chi\left(\Gamma_{i}\right)=0$ for either (then both) $i=1,2$ and $\operatorname{rk}_{k_{i}} \underline{G}_{i}=0$ for both $i=1,2$. Then $\operatorname{sign} \rho^{(2)}\left(\Gamma_{1}\right)=\operatorname{sign} \rho^{(2)}\left(\Gamma_{2}\right)$.

We conjecture that the assumption on $\operatorname{rk}_{k_{i}} \underline{G}_{i}$ is not needed. It would not be needed if [43, Conjecture 1.2] was true, and it is not needed if the fundamental rank of $\underline{G}_{i}$ defined in Section 2 is even [29, Theorem 1.2]. But in our proof, we are using the equality of analytic and cellular $\ell^{2}$-torsion that is, at present, only known if $\Gamma_{i}$ is a cocompact lattice in the Lie group $\prod_{v} \underline{G}_{i}\left(k_{i v}\right)$ where $v$ runs through the infinite places of $k_{i}$. This cocompactness condition is equivalent to $\mathrm{rk}_{k_{i}} \underline{G}_{i}=0$. Interesting examples to which the theorem applies can be found among cocompact lattices in $\operatorname{Spin}(p, q)$ and $\operatorname{Spin}(p-4, q+4)$ for odd integers $p, q \geq 7$ : Combining methods of Aka [3] with Kneser's solution of the congruence subgroup problem for anisotropic spinor groups [37], one can find an arithmetic $\mathbb{Q}$-anisotropic lattice in each group such that the two lattices are profinitely isomorphic. According to Olbrich [48], these have nonzero $\ell^{2}$-torsion. By our theorem, the values have the same sign. 
If $\Gamma$ and $\Lambda$ are of type $(F)$ and $\Lambda$ is residually finite and $\ell^{2}$-acyclic, then we have the product formula $\rho^{(2)}(\Gamma \times \Lambda)=\chi(\Gamma) \rho^{(2)}(\Lambda)$ as proven in [42, Theorem 3.93 (4)]. Hence if $M$ is some closed hyperbolic 3-manifold, then

$$
2 \rho^{(2)}\left(\Gamma_{8,2} \times \pi_{1} M\right)=\rho^{(2)}\left(\Gamma_{4,6} \times \pi_{1} M\right)<0 .
$$

The groups $\Lambda_{i}=\pi_{1} M \times \Gamma_{4-i}$, where $\Gamma_{4-i}$ was introduced below Corollary 1.3, are residually finite and of type $(F)$, and

$$
\rho^{(2)}\left(\Lambda_{1}\right)<0, \quad \rho^{(2)}\left(\Lambda_{2}\right)=0, \quad \rho^{(2)}\left(\Lambda_{3}\right)>0 .
$$

This shows that, as before, Theorem 1.4 has no immediate extension in one way or another.

Since an arithmetic group $\Gamma$ has at most one nonzero $\ell^{2}$-Betti number, the Euler characteristic $\chi(\Gamma)$ encodes the entire reduced $\ell^{2}$-cohomology. The lesser-known Novikov-Shubin invariants $\alpha_{p}(\Gamma)$ capture whether $\Gamma$ additionally possesses unreduced $\ell^{2}$-cohomology. The reader can find an overview in [42, Chapter 2]. In the semisimple and $k$-anisotropic case, our methods imply an even stronger statement on these subtle invariants. To state it, let us introduce the relabeling $\bar{\alpha}_{ \pm q}(\Gamma)=\alpha_{k \pm q}(\Gamma)$, where the symmetric space on which $\Gamma$ acts is either $2 k$ - or $(2 k+1)$-dimensional.

Theorem 1.5. For $i=1,2$, let $k_{i}$ be number fields, let $\underline{G}_{i}$ be semisimple linear algebraic $k_{i}$-groups with $\mathrm{rk}_{k_{i}} \underline{G}_{i}=0$, and let $\Gamma_{i} \leq \underline{G}_{i}$ be arithmetic. Suppose that $\underline{G}_{1}$ and $\underline{G}_{2}$ have a finite congruence kernel and that $\Gamma_{1}$ is profinitely commensurable with $\Gamma_{2}$. Then $\bar{\alpha}_{ \pm q}\left(\Gamma_{1}\right)=\overline{\bar{\alpha}}_{ \pm q}\left(\Gamma_{2}\right)$ for all $q$.

This time, the assumption that $\operatorname{rk}_{k_{i}} \underline{G}_{i}=0$ is likely to be essential because only in the cocompact case do analytic and cellular Novikov-Shubin invariants agree [21], and only the analytic NovikovShubin invariants are entirely governed by the fundamental rank. Compare [28, Theorem 1.4]. Given a semisimple Lie group $G$ with symmetric space $X=G / K$, let us set $n=\operatorname{dim} X$, and let $m=\delta(G)$ be the fundamental rank. For a torsion-free cocompact lattice $\Gamma \leq G$, Olbrich [48, Theorem 1.1.(b)] has shown in the analytic approach that $\alpha_{p}(\Gamma) \neq \infty^{+}$if and only if $p \in\left[\frac{n-m}{2}, \frac{n+m}{2}-1\right]$. Moreover, in this range, we have $\alpha_{p}(\Gamma)=m$. The arithmetic groups $\Gamma_{i}$ are finitely generated linear in characteristic zero and hence have torsion-free subgroups of finite index by Selberg's lemma. Novikov-Shubin invariants are unchanged when passing to commensurable groups [42, Theorem 2.55 (6)]. Since we show in Theorem 2.1 that $m=\delta(G)$ is a profinite invariant for arithmetic subgroups of semisimple groups, Theorem 1.5 follows.

\subsection{Toward S-arithmetic groups and weakening CSP}

In general, we do not know whether our results generalize from arithmetic to $S$-arithmetic groups. However, we can extend our results in special cases. For example, for groups

$$
\Gamma_{i}=\operatorname{Spin}\left(q_{i}\right)\left(\mathbb{Z}\left[S_{i}^{-1}\right]\right)
$$

where $S_{i}$ are finite sets of rational primes and $q_{i}$ are integral quadratic forms such that $\operatorname{Spin}\left(q_{i}\right)$ has a finite $S_{i}$-congruence kernel, we checked that still sign $\chi\left(\Gamma_{1}\right)=\operatorname{sign} \chi\left(\Gamma_{2}\right)$ whenever $\Gamma_{1}$ and $\Gamma_{2}$ are profinitely commensurable. The proof is a case-by-case study invoking the classification of anisotropic quadratic forms over $\mathbb{Q}_{p}$. Interestingly, and as opposed to the arithmetic case, for these $S$-arithmetic groups, it is no longer true that the dimension of the symmetric space is a profinite invariant mod 4. However, if $\Gamma_{1}$ and $\Gamma_{2}$ are profinitely commensurable and $\operatorname{dim} X_{1} \not \equiv \operatorname{dim} X_{2} \bmod 4$, then there always exists a finite prime $p \in S_{1} \cap S_{2}$ such that $\mathrm{rk}_{\mathbb{Q}_{p}} \underline{G}_{1} \not \equiv \mathrm{rk}_{\mathbb{Q}_{p}} \underline{G}_{2}$ mod 2 so that still sign $\chi\left(\Gamma_{1}\right)=\operatorname{sign} \chi\left(\Gamma_{2}\right)$. An example of this behavior is presented in Example 4.9.

Another family of $S$-arithmetic groups for which we can establish profiniteness of the sign of the Euler characteristic is the following: fixing a (higher-rank simple) $\mathbb{Q}$-group $\underline{G}$, non-commensurable but profinitely commensurable $S$-arithmetic groups occur when $\underline{G}$ is considered over varying number fields. Methods due to Aka [2] are used in [30] to show that these groups must be defined over arithmetically 
equivalent number fields $k$ and $l$. This implies profiniteness of $\operatorname{sign} \chi(\Gamma)$ if $S$ contains no places over ramified primes or if $\underline{G}$ splits over $k$ and $l$.

It is unclear if these observations can be extended to general algebraic groups with CSP. Notwithstanding, we can strengthen Theorems 1.1 and 1.4 formally by only requiring that one of the two groups be arithmetic and have CSP.

Theorem 1.6. Let $\underline{G}_{1}$ and $\underline{G}_{2}$ be linear algebraic groups defined over number fields $k_{1}$ and $k_{2}$. Suppose $\underline{G}_{1}$ has finite congruence kernel and that either $\underline{G}_{2}$ is reductive and each $k_{2}$-simple factor of the universal covering of its derived subgroup satisfies the Platonov-Margulis conjecture, or $\underline{G}_{2}$ is not reductive. Let $\Gamma_{1} \leq \underline{G}_{1}$ be arithmetic, and let $\Gamma_{2} \leq \underline{G}_{2}$ be $S$-arithmetic for a finite set of places $S$ of $k_{2}$ containing all the infinite ones.

1. If $\Gamma_{1}$ and $\Gamma_{2}$ are profinitely commensurable, then

$$
\operatorname{sign} \chi\left(\Gamma_{1}\right)=\operatorname{sign} \chi\left(\Gamma_{2}\right)
$$

2. If, in addition, $\operatorname{rk}_{k_{1}} \underline{G}_{1}=\operatorname{rk}_{k_{2}} \underline{G}_{2}=0$ and $\chi\left(\Gamma_{1}\right)=\chi\left(\Gamma_{2}\right)=0$, then

$$
\operatorname{sign} \rho^{(2)}\left(\Gamma_{1}\right)=\operatorname{sign} \rho^{(2)}\left(\Gamma_{2}\right) .
$$

See [49] for an introduction to the Platonov-Margulis conjecture and [52, Appendix A] for a shorter and more up-to-date survey. We note that while this conjecture is still open in some cases, its status is still better than that of the congruence subgroup property; in particular, it is known to hold for inner forms of type $A_{n}$. Unlike Theorem 1.1, the above result can be applied when the $\mathbb{R}$-points of the Weil restriction of $\underline{G}_{2}$ are of real rank one; Theorem 1.1 is not applicable since real and complex hyperbolic lattices often do not have CSP (and are conjectured to never have it).

\subsection{Comments on rank-one groups}

Finally, the question occurs whether the assumption of CSP in Theorem 1.1 can be removed, which by Serre's conjecture should boil down to understanding the case of rank-one simple Lie groups. Taking the classification of rank-one simple real Lie groups into account, the profiniteness of the sign of Euler characteristic or $\ell^{2}$-torsion reduces to the question of profiniteness of the dimension of the symmetric space modulo 4.

However, the techniques used to prove such a statement would by necessity be very different from the rigidity results used in higher rank, except possibly for lattices in the quaternionic hyperbolic spaces and the octonionic hyperbolic plane. There has already been much work on this topic or related topics; some topological profinite invariants for 3-manifold groups are given in [7]. Let us also mention the following results of interest:

1. Recent work of Bridson, McReynolds, Reid, and Spitler [12] shows that profinite rigidity holds for certain Kleinian groups, including the Weeks manifold group. Note that profinite rigidity is generally hard to establish. It is, for example, open whether free groups or, more generally, Fuchsian groups, Kleinian groups, $\operatorname{SL}(n, \mathbb{Z})$, or mapping class groups of closed surfaces are profinitely rigid.

2. The question becomes more accessible if one only asks for profinite rigidity among a certain class of groups. In this vein, Bridson and Reid [13] had previously shown that the figure-eight knot group is a Kleinian group that is profinitely rigid among 3-manifold groups. In general, it is not even known whether Kleinian groups are profinitely rigid among themselves. In fact, it is open whether the volume of hyperbolic 3-manifolds is profinite in the sense that it agrees for two such manifolds whose fundamental groups have isomorphic profinite completions.

3. Fuchsian groups are profinitely rigid among lattices in Lie groups and S-arithmetic groups; this follows from profiniteness invariance of the first $L^{2}$-Betti number, which distinguishes them from lattices in other Lie groups, and the work of Bridson, Conder, and Reid [11] that distinguishes them between themselves. 
4. It follows from the work of Bergeron, Haglund, and Wise [6] and Minasyan and Zalesskii [46] that arithmetic lattices of simple type in $\operatorname{SO}(n, 1)$ (for any $n$ ) are cohomologically good; in particular, their profinite completion knows their virtual cohomological dimension, which equals $n$ for a uniform lattice and $n-1$ for a non-uniform one. It is well-known that Fuchsian groups are good. It also follows from the work of Agol [1] together with that of Minasyan and Zalesskii that lattices in $\mathrm{SO}(3,1)$ are cohomologically good.

5. Recently, M. Stover [58] gave examples, for any $n \geq 2$, of a pair of lattices in $\mathrm{PU}(n, 1)$ that are profinitely isomorphic but not commensurable to each other.

\subsection{Outline}

In Section 2, we establish profinite invariance of the fundamental rank of the associated Lie groups as well as profinite invariance of the dimension of the associated symmetric space mod 4 in the semisimple case. Section 3 then derives the main results as straightforward conclusions from the previous section. In Section 4, we explicitly compute the Euler characteristic of the arithmetic spin groups $\Gamma_{m, n}$.

\section{Profinite invariance of fundamental rank and dimension mod 4}

For better reference, we have chosen to formulate our results in the introduction in terms of number fields. But, as is well known, given a number field $k$ and a linear algebraic $k$-group $\underline{G}$, the restriction of scalars functor $\operatorname{Res}_{\mathbb{Q}}^{k}$ as, for instance, introduced in [49, Section 2.1.2] comes with a natural isomorphism $\operatorname{Res}_{\mathbb{Q}}^{k} \underline{G}(\mathbb{Q}) \cong \underline{G}(k)$ that preserves the notion of an arithmetic subgroup and satisfies, moreover, $C\left(\operatorname{Res}_{\mathbb{Q}}^{k} \underline{G}, \mathbb{Q}\right) \cong C(\underline{G}, k)$ for the congruence kernels. Thus every arithmetic subgroup of a $k$-group is isomorphic to an arithmetic subgroup of a $\mathbb{Q}$-group, and the former has finite congruence kernel if and only if the latter does. These remarks justify that henceforth we will work over $k=\mathbb{Q}$ only. As an outcome of the introduction, we see that the following theorem is the main technical result we need to attack.

Theorem 2.1. Let $\Gamma_{1} \leq \underline{G}_{1}$ and $\Gamma_{2} \leq \underline{G}_{2}$ be arithmetic subgroups of semisimple linear algebraic $\mathbb{Q}$ groups with finite congruence kernel. If $\Gamma_{1}$ is profinitely commensurable with $\Gamma_{2}$, then

1. $\operatorname{dim} X_{1} \equiv \operatorname{dim} X_{2} \bmod 4$ and

2. $\delta\left(G_{1}\right)=\delta\left(G_{2}\right)$.

Here, $X_{i}=G_{i} / K_{i}$ is the symmetric space associated with $\underline{G}_{i}$. It is defined by choosing a maximal compact subgroup $K_{i} \subseteq G_{i}$ of the Lie group $G_{i}=\underline{G}_{i}(\mathbb{R})$. The number

$$
\delta\left(G_{i}\right)=\operatorname{rk}_{\mathbb{C}}\left(\mathcal{L}\left(G_{i}\right) \otimes_{\mathbb{R}} \mathbb{C}\right)-\operatorname{rk}_{\mathbb{C}}\left(\mathcal{L}\left(K_{i}\right) \otimes_{\mathbb{R}} \mathbb{C}\right)
$$

is called the fundamental rank of $X_{i}$, sometimes also known as the deficiency of $G_{i}$. The notation $\mathcal{L}\left(G_{i}\right)$ and $\mathcal{L}\left(K_{i}\right)$ denotes the Lie algebras of the Lie groups $G_{i}$ and $K_{i}$.

The rough outline of the proof of Theorem 2.1 is as follows. We first show that under the assumption of CSP and strong approximation, the profinite commensurability of $\Gamma_{1}$ and $\Gamma_{2}$ implies that the Lie algebras of the $p$-adic analytic groups $\underline{G}_{1}\left(\mathbb{Q}_{p}\right)$ and $\underline{G}_{2}\left(\mathbb{Q}_{p}\right)$ are isomorphic for all finite primes $p$ (Proposition 2.4). Weil's product formula expresses the signature of a rational quadratic form mod 8 in terms of Gaussian sums associated with the $\mathbb{F}_{p}$-reductions of the form. Applying this formula to the Killing forms of the Lie algebras of $\underline{G}_{1}$ and $\underline{G}_{2}$, we can conclude that $\operatorname{dim} X_{1} \equiv \operatorname{dim} X_{2} \bmod 4$ (Proposition 2.5). To show that $\delta\left(G_{1}\right)=\bar{\delta}\left(G_{2}\right)$, we first explain that if $\underline{G}_{1}\left(\mathbb{Q}_{p}\right) \cong \underline{G}_{2}\left(\mathbb{Q}_{p}\right)$ for all $p$, then of necessity $\underline{G}_{1} \times_{\mathbb{Q}} \mathbb{R}$ and $\underline{G}_{2} \times \mathbb{Q} \mathbb{R}$ are inner forms of one another (Proposition 2.7 ). Therefore, if we fix an isomorphism $\varphi: \underline{G}_{1} \times \mathbb{Q} \mathbb{C} \rightarrow \underline{G}_{2} \times \mathbb{Q} \mathbb{C}$, and if $\tau_{i}$ denotes the complex conjugation satisfying $\underline{G}_{i}(\mathbb{C})^{\tau_{i}}=\underline{G}_{i}(\mathbb{R})$, then $\tau_{1}$ and $\varphi^{-1} \tau_{2} \varphi$ are conjugate by an inner automorphism. In that case, the maximal compact subgroups of $\underline{G}_{i}(\mathbb{R})$ have the the same rank as we verify in Proposition 2.9 so that equality of fundamental ranks follows. 
To begin with, we verify that in a typical situation, isomorphisms of products of $p$-adic Lie groups must be factor-wise. The notation ' $\leq_{o}$ ' and ' $\leq_{c}$ ' indicates open and closed subgroups, respectively.

Lemma 2.2. Let $G$ be a profinite group with open subgroups $A, B \leq_{o} G$. Suppose that

$$
A=\prod_{p} G_{p} \quad \text { and } \quad B=\prod_{p} H_{p}
$$

for certain p-adic analytic groups $G_{p}, H_{p} \leq_{c} G$, where the product runs over all prime numbers. Then $H_{p}$ and $G_{p}$ are virtually isomorphic for all primes $p$. In particular, the Lie algebras $\mathcal{L}\left(H_{p}\right)$ and $\mathcal{L}\left(G_{p}\right)$ are $\mathbb{Q}_{p}$-isomorphic.

Proof. Let $p$ be a prime. We may assume that $\operatorname{dim}\left(G_{p}\right) \geq \operatorname{dim}\left(H_{p}\right)$. After possibly shrinking $B$, we may assume $B \subseteq A=G$. Let $\pi: G \rightarrow G_{p}$ denote the projection homomorphism. For every prime $\ell \neq p$, the image $\pi\left(H_{\ell}\right)$ is $p$-adic and $\ell$-adic analytic and hence a finite group. Moreover, let $U_{p} \unlhd_{o} G_{p}$ be an open normal uniform pro- $p$ subgroup (the existence follows from [18, Corollary 8.34]). Recall that such a group is torsion-free (see [18, Theorem 4.5]). Thus $\pi\left(H_{\ell}\right) \cap U_{p}=\{1\}$ since it is finite and torsion-free.

Now we consider the homomorphism $\bar{\pi}: G \rightarrow G_{p} / U_{p}$ composed from $\pi$ and the canonical factor map $G_{p} \rightarrow G_{p} / U_{p}$. Since $\bar{\pi}$ is continuous, its kernel is open, and there is a finite set of primes $S$ (with $p \in S$ ) such that

$$
\bar{\pi}\left(\prod_{\ell \notin S} H_{\ell}\right)=\{1\} .
$$

It follows that $\pi\left(\prod_{\ell \neq p} H_{\ell}\right)$ is finite. However, the homomorphism $\pi$ is surjective, and we deduce that $\pi\left(H_{p}\right)$ is an open subgroup of $G_{p}$.

Choose an open normal uniform pro- $p$ subgroup $V_{p} \unlhd_{o} H_{p}$ such that $\pi\left(V_{p}\right) \subseteq U_{p}$. Since $V_{p}$ is finitely generated powerful (see [18, Definition 2.1]) and $U_{p}$ is torsion-free, we deduce that $\pi\left(V_{p}\right)$ is a finitely generated, powerful, torsion-free pro- $p$ group. By [18, Theorem 4.5] we get that $\pi\left(V_{p}\right)$ is a uniform subgroup in $U_{p}$, and as it is also open, we have $\operatorname{dim} \pi\left(V_{p}\right)=\operatorname{dim} U_{p}, \operatorname{sodim} V_{p} \geq \operatorname{dim} U_{p}$. By assumption, $\operatorname{dim}\left(V_{p}\right) \leq \operatorname{dim}\left(U_{p}\right)$, and we conclude that the dimensions are equal and that $\left.\pi\right|_{V_{p}}$ is an isomorphism onto its image.

Remark 2.3. For an affine group scheme $\underline{H}$ over a commutative ring $R$, the Lie algebra (functor) will be denoted by $\operatorname{Lie}(\underline{H})$. For a Lie group $U$ over a complete valued field $k$, such as $\mathbb{R}, \mathbb{C}$ or $\mathbb{Q}_{p}$, the associated $k$-Lie algebra will be denoted by $\mathcal{L}(U)$. Recall that, if $\underline{G}$ is a linear algebraic group over $k$, then $\underline{G}(k)$ is a $k$-analytic Lie group, and

$$
\operatorname{Lie}(\underline{G})(k) \cong \mathcal{L}(\underline{G}(k)) .
$$

A little more generally than necessary, we will now see that assuming CSP and strong approximation, profinitely commensurable $S$-arithmetic subgroups lie in algebraic groups whose Lie algebras become isomorphic when completing the field outside $S$.

Proposition 2.4. Let $S_{1}$ and $S_{2}$ be finite sets of places of $\mathbb{Q}$ containing the infinite one, and let $\underline{G}_{1}$ and $\underline{G}_{2}$ be algebraic $\mathbb{Q}$-groups. Assume $\underline{G}_{i}$ has finite $S_{i}$-congruence kernel and strong approximation with

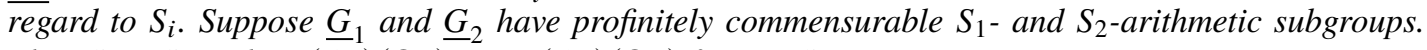
Then $S_{1}=S_{2}$ and $\operatorname{Lie}\left(\underline{G}_{1}\right)\left(\mathbb{Q}_{p}\right) \cong \operatorname{Lie}\left(\underline{G}_{2}\right)\left(\mathbb{Q}_{p}\right)$ for $p \notin S_{1}$.

Proof. Choose $S_{i}$-arithmetic subgroups $\Gamma_{i} \subseteq \underline{G}_{i}(\mathbb{Q})$ such that $\widehat{\Gamma}_{1} \cong \widehat{\Gamma}_{2}$. Since the congruence kernels are finite, we can pass to finite index subgroups if need be, to assume that $\widehat{\Gamma}_{i}$ is (isomorphic to) the closure of $\Gamma_{i}$ in $\underline{G}_{i}\left(\mathbb{A}_{S_{i}}\right)$. Here, $\left(\mathbb{A}_{S_{i}}\right)$ denotes the ring of $\left(S_{i}\right)$-adeles, that is, the restricted product $\left(\prod_{p \notin S_{i}}^{\prime} \mathbb{Q}_{p}\right)$ consisting of all elements in the product with all but finitely many coordinates in $\left(\mathbb{Z}_{p}\right)$. Strong approximation implies that $\left(\widehat{\Gamma}_{i}\right)$ is an open subgroup of $\left(\underline{G}_{i}\left(\mathbb{A}_{S_{i}}\right)\right)$. In particular, it has an open 
subgroup that is isomorphic to a product $\prod_{p \notin S_{i}} U_{p}^{(i)}$ for certain open compact subgroups $U_{p}^{(i)} \leq_{o}$ $\underline{G}_{i}\left(\mathbb{Q}_{p}\right)$. So clearly, $S_{1}=S_{2}$ and Lemma 2.2 and Remark 2.3 complete the proof.

The following is a variation of an observation of Rohlfs and Speh [54, Lemma 2.5]; see also [35, Lemma 4].

Proposition 2.5. Let $\underline{G}_{1}$ and $\underline{G}_{2}$ be semisimple linear algebraic groups over $\mathbb{Q}$ with associated symmetric spaces $X_{1}=G_{1} / K_{1}$ and $X_{2}=G_{2} / K_{2}$. If $\operatorname{Lie}(G)\left(\mathbb{Q}_{p}\right) \cong \operatorname{Lie}(H)\left(\mathbb{Q}_{p}\right)$ for all $p$, then

$$
\operatorname{dim} X_{1} \equiv \operatorname{dim} X_{2} \bmod 4 .
$$

Proof. We note that $\operatorname{Lie}\left(\underline{G}_{i}\right)(k)=k \otimes_{\mathbb{Q}} \operatorname{Lie}\left(\underline{G}_{i}\right)(\mathbb{Q})$ for every extension field $k$ of $\mathbb{Q}$. The Killing forms $\beta_{i}$ on $\operatorname{Lie}\left(\underline{G}_{i}\right)(\mathbb{Q})$ are non-degenerate symmetric bilinear forms defined over $\mathbb{Q}$. The Cartan decomposition implies that $\beta_{i}$ has signature $\left(\operatorname{dim}\left(X_{i}\right), \operatorname{dim}\left(K_{i}\right)\right)$ as a form on $\operatorname{Lie}\left(\underline{G}_{i}\right)(\mathbb{R})$.

The Killing form is completely determined by the Lie algebra structure; hence the quadratic spaces $\left(\operatorname{Lie}\left(\underline{G}_{1}\right)\left(\mathbb{Q}_{p}\right), \beta_{1}\right)$ and $\left(\operatorname{Lie}\left(\underline{G}_{2}\right)\left(\mathbb{Q}_{p}\right), \beta_{2}\right)$ are isometric for every prime number $p$. Weil's product formula implies that $\operatorname{dim}\left(X_{1}\right)-\operatorname{dim}\left(K_{1}\right) \equiv \operatorname{dim}\left(X_{2}\right)-\operatorname{dim}\left(K_{2}\right) \bmod 8$; see [55, Corollary 8.2]. Let $d=\operatorname{dim}\left(G_{1}\right)=\operatorname{dim}\left(G_{2}\right)$; then $d=\operatorname{dim}\left(X_{1}\right)+\operatorname{dim}\left(K_{1}\right)=\operatorname{dim}\left(X_{2}\right)+\operatorname{dim}\left(K_{2}\right)$, and we deduce that

$$
2 \operatorname{dim}\left(X_{1}\right) \equiv \operatorname{dim}\left(X_{1}\right)-\operatorname{dim}\left(K_{1}\right)+d \equiv 2 \operatorname{dim}\left(X_{2}\right) \bmod 8 .
$$

Definition 2.6. Let $\underline{G}_{1}, \underline{G}_{2}$ be linear algebraic groups over a field $k$ of characteristic 0 . We say that $\underline{G}_{2}$ is an inner form of $\underline{G}_{1}$ if there is an isomorphism $\varphi: \underline{G}_{1} \times_{k} \bar{k} \rightarrow \underline{G}_{2} \times_{k} \bar{k}$ (where $\bar{k}$ is the algebraic closure of $k$ ) such that $\varphi^{-1} \sigma \varphi \sigma^{-1}$ is an inner automorphism of $\underline{G}_{1} \overline{\times}_{k} \bar{k}$ for all $\sigma \in \operatorname{Gal}(\bar{k} / k)$.

Proposition 2.7. Let $S$ be a finite set of places of $\mathbb{Q}$ containing the Archimedean place. Let $\underline{G}_{1}$ and $\underline{G}_{2}$ be simply connected semisimple algebraic groups over $\mathbb{Q}$ such that $\operatorname{Lie}\left(\underline{G}_{1}\right)\left(\mathbb{Q}_{p}\right) \cong \operatorname{Lie}\left(\underline{G}_{2}\right)\left(\mathbb{Q}_{p}\right)$ for all $p \notin S$. Then $\underline{G}_{2} \times_{\mathbb{Q}} \mathbb{R}$ is an inner form of $\underline{G}_{1} \times_{\mathbb{Q}} \mathbb{R}$.

Proof. Our goal is to reach a contradiction between $\underline{G}_{i} \times_{\mathbb{Q}} \mathbb{R}$ being outer forms of each other and the hypothesis that $G_{i} \times \mathbb{Q} \mathbb{Q}_{p}$ is locally isomorphic for almost all $p$. The latter implies, in particular, that $\operatorname{Lie}\left(\underline{G}_{1}\right)(L) \cong \operatorname{Lie}\left(\underline{G}_{2}\right)(L)$ for some finite Galois extension $L / \mathbb{Q}$. Since simply connected semisimple groups are determined up to isomorphism by their Lie algebras, we deduce

$$
\underline{G}_{1} \times_{\mathbb{Q}} L \cong \underline{G}_{2} \times_{\mathbb{Q}} L .
$$

Without loss of generality, we may assume, after possibly passing to a larger field $L$, that $\underline{G}_{1} \times_{\mathbb{Q}} L$ is split. Note that for any extension field $L^{\prime}$ of $L$, the group $\underline{G}_{1} \times_{\mathbb{Q}} L^{\prime}$ is split and simply connected, and there is an exact sequence

$$
1 \longrightarrow \operatorname{Ad}\left(\underline{G}_{1} \times_{\mathbb{Q}} L^{\prime}\right) \longrightarrow \operatorname{Aut}\left(\underline{G}_{1} \times_{\mathbb{Q}} L^{\prime}\right) \stackrel{\pi_{L^{\prime}}}{\longrightarrow} \operatorname{Aut}(\operatorname{Dyn}(\Phi)) \longrightarrow 1
$$

where $\operatorname{Dyn}(\Phi)$ denotes the Dynkin diagram of the root system $\Phi$ of $\underline{G}_{1} \times_{\mathbb{Q}} L$ and $\operatorname{Aut}(\operatorname{Dyn}(\Phi))$; see [39, (25.16)]. In addition, for every Galois extension $L_{1} / L_{2}$, where $L_{1}$ contains $L$, the short exact sequence is Galois equivariant, where the action of the Galois group $\operatorname{Gal}\left(L_{1} / L_{2}\right)$ on $\operatorname{Aut}(\operatorname{Dyn}(\Phi))$ is the one induced from the action of $\operatorname{Gal}(L / \mathbb{Q})$.

We choose an isomorphism $\varphi: \underline{G}_{1} \times_{\mathbb{Q}} L \rightarrow \underline{G}_{2} \times_{\mathbb{Q}} L$ and consider the corresponding 1-cocycle defined by $a_{\sigma}=\varphi^{-1} \sigma \varphi \sigma^{-1} \in \operatorname{Aut}\left(\underline{G}_{1} \times \mathbb{Q} L\right)$ for all $\sigma \in \operatorname{Gal}(L / \mathbb{Q})$. The associated non-abelian cohomology class in $H^{1}\left(\operatorname{Gal}(L / \mathbb{Q})\right.$, Aut $\left.\left(\underline{G}_{1} \times \mathbb{Q} L\right)\right)$ will be denoted by $[a]$ and is independent of the choice of $\varphi$. Pick an embedding $\iota: L \rightarrow \mathbb{C}$. We note that $\iota$ induces a homomorphism $\iota^{*}: \operatorname{Gal}(\mathbb{C} / \mathbb{R}) \rightarrow \operatorname{Gal}(L / \mathbb{Q})$ of groups.

From now on, we suppose that $\underline{G}_{2} \times \mathbb{Q} \mathbb{R}$ is not an inner form of $\underline{G}_{1} \times \mathbb{Q} \mathbb{R}$. In this case, the image $\iota(L)$ is not contained in $\mathbb{R}$, since otherwise $\underline{G}_{1} \times \mathbb{Q}$ and $\underline{G}_{2} \times \mathbb{Q} \mathbb{R}$ are isomorphic. The long exact sequence 
associated with (2.1) contains the following segment:

$$
\begin{aligned}
& H^{1}\left(\operatorname{Gal}(\mathbb{C} / \mathbb{R}), \operatorname{Ad}\left(\underline{G}_{1} \times_{\mathbb{Q}} \mathbb{C}\right)\right) \longrightarrow H^{1}\left(\operatorname{Gal}(\mathbb{C} / \mathbb{R}), \operatorname{Aut}\left(\underline{G}_{1} \times_{\mathbb{Q}} \mathbb{C}\right)\right) \\
& \stackrel{\pi_{\mathbb{C}}}{\longrightarrow} H^{1}(\operatorname{Gal}(\mathbb{C} / \mathbb{R}), \operatorname{Aut}(\operatorname{Dyn}(\Phi))),
\end{aligned}
$$

which shows that the class $\pi_{\mathbb{C}}\left(\iota^{* *}[a]\right) \in H^{1}(\operatorname{Gal}(\mathbb{C} / \mathbb{R})$, Aut $(\operatorname{Dyn}(\Phi)))$ is non-trivial. ${ }^{1}$ Let $\tau \in$ $\operatorname{Gal}(\mathbb{C} / \mathbb{R})$ denote complex conjugation. By Chebotarev's density theorem (see [47, Theorem 13.4]), there are a prime number $p \notin S$ and a prime ideal $\mathfrak{p} \subseteq \mathcal{O}_{L}$ lying over $p$ such that $L_{\mathfrak{p}} / \mathbb{Q}_{p}$ is an unramified quadratic extension and the image of $\operatorname{Gal}\left(L_{\mathfrak{p}} / \mathbb{Q}_{p}\right) \rightarrow \operatorname{Gal}(L / \mathbb{Q})$ is $\left\langle\iota^{*} \tau\right\rangle$. Let $j: L \rightarrow L_{\mathfrak{p}}$ denote the inclusion map. Naturality of the above long exact sequence shows that the cohomology class $\pi_{L_{\mathfrak{p}}}\left(j^{* *}([a])\right) \in H^{1}\left(\operatorname{Gal}\left(L_{\mathfrak{p}} / \mathbb{Q}_{p}\right)\right.$, Aut $\left.(\operatorname{Dyn}(\Phi))\right)$, and hence also $j^{* *}([a]) \in$ $H^{1}\left(\operatorname{Gal}\left(L_{\mathfrak{p}} / \mathbb{Q}_{p}\right), \operatorname{Aut}\left(\underline{G}_{1} \times_{\mathbb{Q}} L_{\mathfrak{p}}\right)\right)$ is non-trivial. By [24, XXIV 7.3.1 (iii)], the natural map

$$
d: \operatorname{Aut}_{L_{\mathfrak{p}}}\left(\underline{G}_{1} \times_{\mathbb{Q}} L_{\mathfrak{p}}\right) \rightarrow \operatorname{Aut}_{L_{\mathfrak{p}}-\operatorname{Lie}}\left(\operatorname{Lie}\left(\underline{G}_{1}\right)\left(L_{\mathfrak{p}}\right)\right)
$$

is an isomorphism. It follows that the $\mathbb{Q}_{p}$-Lie algebras $\operatorname{Lie}\left(\underline{G}_{1}\right)\left(\mathbb{Q}_{p}\right)$ and $\operatorname{Lie}\left(\underline{G}_{2}\left(\mathbb{Q}_{p}\right)\right)$ are outer forms of each other; in particular, they are not isomorphic, which yields a contradiction since we assumed that $p \notin S$.

The next proposition will show that inner forms of real Lie groups have maximal compact subgroups of the same rank. We will use in this context that inner forms can be realized as the fixed-point sets of conjugate involutions on the complexification (viewed as real Lie group). In the course of the proof, we will need the following lemma, which should be standard but for which we could not find a reference.

Lemma 2.8. Let $K$ be a compact Lie group, and let $\tau \in \operatorname{Aut}(K)$ be an automorphism of order two. There is a maximal torus $T \subseteq K$ that is $\tau$-stable: that is, $\tau(T)=T$.

Proof. Since $\tau\left(K^{0}\right)=K^{0}$ and all tori are contained in the connected component $K^{0}$, we may assume that $K$ is connected. Let $\mathfrak{f}$ denote the Lie algebra of $K$. The correspondence between maximal tori of $K$ and maximal abelian subalgebras of $\mathfrak{f}$ (see also $[36,4.30]$ ) shows that it suffices to prove the corresponding result for Lie algebras.

We decompose $\mathfrak{f}$ as

$$
\mathfrak{l}=\mathfrak{l}^{\tau} \oplus \mathfrak{f}_{-},
$$

where $\mathfrak{f}^{\tau}$ is the subalgebra of $\tau$-invariant elements and $\mathfrak{f}_{-}$is the $(-1)$-eigenspace of $\tau$. Let $\mathfrak{a} \subseteq \mathfrak{f}^{\tau}$ be a maximal abelian subalgebra of $\mathfrak{f}^{\tau}$. Let $\mathfrak{c}(\mathfrak{a})=\{X \in \mathfrak{f} \mid[X, Y]=0$ for all $Y \in \mathfrak{a}\}$ be the centralizer of $\mathfrak{a}$.

Choose a subspace $\mathfrak{b} \subset \mathfrak{f}_{-} \cap \mathfrak{c}(\mathfrak{a})$ that is maximal abelian: that is, it is maximal with the property $[\mathfrak{b}, \mathfrak{b}]=0$. We define $\mathfrak{t}=\mathfrak{a}+\mathfrak{b}$, and we will show that $\mathfrak{t}$ is a maximal abelian subalgebra of $\mathfrak{f}$. Clearly, $[\mathfrak{t}, \mathfrak{t}]=[\mathfrak{a}, \mathfrak{a}]+[\mathfrak{a}, \mathfrak{b}]+[\mathfrak{b}, \mathfrak{b}]=0$ shows that $t$ is abelian. Now suppose that $\mathfrak{h} \supseteq \mathfrak{a}$ is a larger abelian subalgebra. Since $\mathfrak{a} \subseteq \mathfrak{h}$, the algebra $\mathfrak{h}$ lies in $\mathfrak{c}(\mathfrak{a})$. In particular, $\mathfrak{h} \cap \mathfrak{f}^{\tau}=\mathfrak{a}$ since $\mathfrak{a}$ is maximal abelian in $\mathfrak{f}^{\tau}$. Similarly, $\mathfrak{h} \cap \mathfrak{f}_{-}=\mathfrak{b}$ since $\mathfrak{b}$ was maximal abelian in $\mathfrak{c}(\mathfrak{a}) \cap \mathfrak{f}_{-}$. Let $X \in \mathfrak{h}$, and write $X=X_{+}+X_{-}$ with $X_{+} \in \mathfrak{l}^{\tau}$ and $X_{-} \in \mathfrak{f}_{-}$. For all $Z \in \mathfrak{a}$, one has

$$
0=[X, Z]=\left[X_{+}, Z\right]+\left[X_{-}, Z\right] \in \mathfrak{f}^{\tau} \oplus \mathfrak{f}_{-},
$$

and therefore $X_{+} \in \mathfrak{c}(\mathfrak{a}) \cap \mathfrak{f}^{\tau}=\mathfrak{a} \subseteq \mathfrak{h}$, so $X_{-} \in \mathfrak{f}-\cap \mathfrak{h}=\mathfrak{b}$. It follows that $\mathfrak{h}=\mathfrak{a}+\mathfrak{b}=\mathfrak{t}$. Finally, we note that $\mathrm{t}$ is $\tau$-stable since $\mathfrak{a}$ and $\mathfrak{b}$ are contained in eigenspaces of $\tau$. We conclude by taking $T=\exp (\mathfrak{t})$.

Proposition 2.9. Let $G$ be a connected real Lie group with finite center, and let $\sigma, \tau$ be two automorphisms of order two. Suppose that $\sigma=\operatorname{int}(g) \circ \tau$ for some $g \in G$. If the fixed-point groups $G^{\tau}$ and $G^{\sigma}$ are connected, then their maximal compact subgroups have the same complex rank.

\footnotetext{
${ }^{1}$ We use a double star $\iota^{* *}$ to denote the pull-back by the homomorphism $\iota^{*}: \operatorname{Gal}(\mathbb{C} / \mathbb{R}) \rightarrow \operatorname{Gal}(L / \mathbb{Q})$ induced from the field embedding $\iota$.
} 
Proof. We first want to reduce to the case where $g \in K$ for some maximal compact subgroup $K$ in $G$. Let $Z \subseteq G$ be the center; we need to find a $k \in K$ such that $k \tau(k) \in Z$ (so $\operatorname{int}(k) \circ \tau$ has order 2) and there exists $h \in G$ such that $h k \tau(h)^{-1} \in g Z$ (so $G^{\sigma^{\prime}}$ is conjugated by $h$ to $G^{\sigma}$, where $\sigma^{\prime}=\operatorname{int}(k) \circ \tau$ ). Let

$$
\widetilde{G}=G \rtimes_{\tau} \mathbb{Z} / 2 .
$$

Let $\widetilde{K}$ be a maximal compact subgroup of $\widetilde{G}$ containing $(1, \tau)$. We have that $K:=\widetilde{K} \cap G$ is a maximal compact subgroup of $G$, as follows, for example, from [26, Theorem 14.1.3]. In addition, we note that $K$ is $\tau$-stable, which we will use later in the proof. We now define the group

$$
\widehat{G}=(G / Z) \rtimes_{\tau} \mathbb{Z} / 2 .
$$

As $g \tau(g)=1$, the element $(g Z, \tau) \in \widehat{G}$ has order 2. It is contained in a maximal subgroup of $\widehat{G}$; hence (since any compact subgroup of $\widehat{G}$ is contained in a conjugate of $K / Z \rtimes \mathbb{Z} / 2$ ) there exists $h \in G$ so that $(h Z, 1)^{-1}(g Z, \tau)(h Z, 1) \in K / Z \rtimes \mathbb{Z} / 2$. This means exactly that there exists $k \in K$ such that $h k \tau(h)^{-1} \in g Z$; this completes the reduction to $g \in K$.

From now on, it suffices to consider $K$ instead of $G$. We need to show that $K^{\tau}$ and $K^{\sigma}$ have the same rank. The centralizer $C_{K}(g)$ is a closed subgroup of $K$. Since $\tau(g) \in g^{-1} Z$, the centralizer $C_{K}(g)$ is stable under $\tau$ (and hence also $\sigma$ ). Since $g$ is contained in a maximal torus of $K$ (for example, [36, 4.36]), the maximal tori in $C_{K}(g)$ are maximal in $K$. By Lemma 2.8, we find a maximal torus $T \subseteq C_{K}(g)$ that is $\tau$-stable. In fact, $g \in T$. This follows from Theorem 4.50 in [36] using that $g$ centralizes $T$, the group $K$ is connected, and $T$ is maximal in $K$.

We can write $g=g_{0} h_{0}$ for elements $g_{0}, h_{0} \in T$ that satisfy $\tau\left(g_{0}\right)=g_{0}$ and $\tau\left(h_{0}\right)=h_{0}^{-1}$ : this follows immediately from the eigenspace decomposition of the Lie algebra $t$ with respect to $\tau$, and the fact that the exponential map exp $: \mathrm{t} \rightarrow T$ is onto and $\tau$-equivariant. For later use, we further write $h_{0}=h^{2}$ for some $h$ with $\tau(h)=h^{-1}$. Observe that $g \tau(g)=g_{0}^{2} \in Z$. The automorphism $\sigma^{\prime}=\operatorname{int}\left(g_{0}\right) \circ \tau$ satisfies $\sigma \circ \operatorname{int}(h)=\operatorname{int}(h) \circ \sigma^{\prime}$; and, as above, conjugation by $h$ provides an isomorphism of the fixed-point groups $K^{\sigma^{\prime}}$ and $K^{\sigma}$. This means we may assume that $g=g_{0}$ and $\tau(g)=g$. In other words, we assume that $g \in K^{\tau} \cap K^{\sigma}$. Since $K^{\tau}$ and $K^{\sigma}$ are connected, the centralizer of any element contains a maximal torus. Finally, we observe that

$$
C_{K}(g) \cap K^{\sigma}=K^{\tau} \cap K^{\sigma}=K^{\tau} \cap C_{K}(g) .
$$

This means a maximal torus of $K^{\tau} \cap K^{\sigma}$ is maximal in both fixed-point groups.

Proof of Theorem 2.1. Recall that we are in the following situation: $\Gamma_{i}$ are profinitely commensurable arithmetic subgroups in the $\mathbb{Q}$-points of two semisimple $\mathbb{Q}$-groups $\underline{G}_{i}, i=1,2$. Moreover, the congruence kernel of each $\underline{G}_{i}$ is finite. To simplify notation, we will assume that $\Gamma_{i}$ are profinitely isomorphic (since taking profinitely isomorphic finite-index subgroups in each does not change the hypotheses).

We start by reducing to the case where $\underline{G}_{i}$ have no $\mathbb{Q}$-factor whose $\mathbb{R}$-points are compact. To do so, let $\underline{G}_{i}=\underline{H}_{i} \times \underline{K}_{i}$, where $\underline{H}_{i}$ satisfies this hypothesis and $\underline{K}_{i} \times_{\mathbb{Q}} \mathbb{R}$ is compact. Let $d$ be the largest of the minimal dimensions of a $\mathbb{Q}$-rational representation of $\underline{G}_{i}$, and let $\Phi_{i}$ be the set of all morphisms $\Gamma_{i} \rightarrow \mathrm{GL}_{k}(\mathbb{Z} / 3)$ for $2 \leq k \leq d$. Then the subgroups $\Gamma_{i}^{\prime}=\bigcap_{\varphi \in \Phi_{i}} \operatorname{ker}(\varphi)$ are profinitely isomorphic. By Minkowski's theorem, they are torsion-free. As $\underline{K}_{i}(\mathbb{Q}) \cap \Gamma_{i}$ is finite, we get that $\Gamma_{i}^{\prime}$ is isomorphic to an arithmetic subgroup of $\underline{H}_{i}(\mathbb{Q})$.

Similarly, we can reduce to the case where $\underline{G}_{i}$ are simply connected: let $\widetilde{G}_{i}$ be the simply connected covers of $\underline{G}_{i}$, and let $n$ be the largest between the minimal indices of a subgroup of $\Gamma_{i}$ lifting to $\widetilde{G}_{i}$. Taking $\Gamma_{i}^{\prime}$ to be the intersection of all subgroups in $\Gamma_{i}$ of index at most $n$, we get two groups $\Gamma_{1}^{\prime}, \Gamma_{2}^{\prime}$ that are profinitely isomorphic and also isomorphic to arithmetic subgroups in $\underline{G}_{1}(\mathbb{Q}), G_{2}(\mathbb{Q})$, respectively.

These new $\underline{G}_{i}$ satisfy the hypotheses of the Kneser-Platonov theorem [49, Theorem 7.12], and it follows that they have strong approximation. We note that both replacement procedures above preserve the property of having a finite congruence kernel, so we can apply to $\underline{G}_{i}$ Proposition 2.4 to deduce that 
$\operatorname{Lie}\left(\underline{G}_{1}\right)\left(\mathbb{Q}_{p}\right) \cong \operatorname{Lie}\left(\underline{G}_{2}\right)\left(\mathbb{Q}_{p}\right)$ for every prime $p$. Applying Proposition 2.5 , we subsequently conclude that $\operatorname{dim}\left(X_{1}\right) \equiv \operatorname{dim}\left(X_{2}\right) \bmod 4$.

Proposition 2.7 implies that the group $\underline{G}_{2} \times \mathbb{Q} \mathbb{R}$ is an inner form of $\underline{G}_{1} \times \mathbb{Q} \mathbb{R}$. Hence an isomorphism $\varphi: \underline{G}_{1} \times_{\mathbb{Q}} \mathbb{C} \rightarrow \underline{G}_{2} \times_{\mathbb{Q}} \mathbb{C}$ can be chosen so that $\varphi^{-1} \tau_{2} \varphi \tau_{1}^{-1}=\operatorname{int}(g)$ for some $g \in \underline{G}_{1}(\mathbb{C})$, where $\tau_{i}$ denotes the involution on $\underline{G}_{i}(\mathbb{C})$ induced by complex conjugation, so that $\underline{G}_{i}(\mathbb{C})^{\tau_{i}}=\underline{G}_{i}(\mathbb{R})$. Setting $\sigma=\varphi^{-1} \tau_{2} \varphi$, we have $\sigma=\operatorname{int}(g) \circ \tau_{1}$ and $\underline{G}_{1}(\mathbb{C}) \sigma \cong \underline{G}_{2}(\mathbb{R})$. Since the groups $\underline{G}_{i}$ are simply connected, the real Lie groups $\underline{G}_{i}(\mathbb{R})$ are connected; see [49, Proposition 7.6]. By Proposition 2.9, we have that $\operatorname{rk}_{\mathbb{C}}\left(\mathcal{L}\left(K_{2}\right) \otimes \mathbb{C}\right)=\operatorname{rk}_{\mathbb{C}}\left(\mathcal{L}\left(K_{1}\right) \otimes \mathbb{C}\right)$, and as $\delta\left(\underline{G}_{i}(\mathbb{R})\right)=\operatorname{rk}_{\mathbb{C}}\left(\operatorname{Lie}\left(\underline{G}_{i}\right)(\mathbb{C})\right)-\operatorname{rk}_{\mathbb{C}}\left(\mathcal{L}\left(K_{i}\right) \otimes \mathbb{C}\right)$, we can conclude that $\delta\left(G_{1}\right)=\delta\left(G_{2}\right)$.

\section{Conclusion of main results}

We prove the main result, Theorem 1.1, and the slightly strengthened version, Theorem 1.6. Most of what we need for the semisimple case is contained in Theorem 2.1. In this section, we put everything together and describe the reduction to the semisimple case.

Lemma 3.1. Let $\underline{G}$ be a semisimple linear algebraic $\mathbb{Q}$-group with finite congruence kernel, and let $\Gamma \leq \underline{G}(\mathbb{Q})$ be an arithmetic subgroup. Then $\widehat{\Gamma}$ has no (topologically) finitely generated infinite closed normal solvable subgroup.

Proof. The desired property of $\widehat{\Gamma}$ stays unchanged by passing to finite index subgroups. The group $\underline{G}$ is an almost direct product of simple $\mathbb{Q}$-groups. A finite index subgroup of $\Gamma$ is an arithmetic subgroup of the product of simple $\mathbb{Q}$-factors whose $\mathbb{R}$-points are non-compact. Moreover, the latter product has a finite congruence kernel [51, p. 400]. So we may and will assume that $\underline{G}$ contains no $\mathbb{Q}$-simple (almost) factor whose $\mathbb{R}$-points are compact. By an observation of Serre, $\underline{G}$ is simply connected [57, $1.2 \mathrm{c})]$. Hence $\underline{G}$ satisfies strong approximation [49, Theorem 7.12]. Since the congruence kernel of $\underline{G}$ is finite, we can assume, by passing once more to a finite index subgroup, that $\widehat{\Gamma}$ is embedded into $\prod_{p} \underline{G}\left(\mathbb{Q}_{p}\right)$. By strong approximation, $\widehat{\Gamma}$ is a compact open subgroup of $\prod_{p} U_{p}$, where each $U_{p}<\underline{G}\left(\mathbb{Q}_{p}\right)$ is a compact open subgroup. Let $\mathrm{pr}_{p}$ be the projection from the product to $U_{p}$. Let $N$ be a finitely generated closed normal solvable subgroup of $\widehat{\Gamma}$. We have to show that $N$ is finite.

If $\operatorname{pr}_{p}(N)$ was infinite for some prime, its Lie subalgebra would be a non-trivial solvable ideal in $\mathcal{L}\left(\underline{G}\left(\mathbb{Q}_{p}\right)\right)$, contradicting semisimplicity of $\underline{G}$. Thus $F_{p}:=\operatorname{pr}_{p}(N)$ is finite for every prime $p$.

We know that $\Gamma$ is a subset of $U_{p} \subseteq \underline{G}\left(\mathbb{Q}_{p}\right)$, and it is Zariski dense in $\underline{G}$ by [44, Proposition (3.2.11)]. Since the finite - in particular, algebraic — subgroup $F_{p}$ of $\underline{G}$ is normalised by the Zariski dense set $\Gamma$, we conclude that $F_{p}$ is a normal subgroup of $\underline{G}$ [45, Proposition 1.38]. Thus it is contained in the center of $\underline{G}$ by semisimplicity. Since there exist embeddings $\underline{G}\left(\mathbb{Q}_{p}\right) \subset \underline{G}(\mathbb{C})$ for all $p$, there is $e \in \mathbb{N}$ such that $F_{p}$ is abelian with exponent $e$ for every prime $p$. In particular, $N$ is abelian with exponent $e$. Since it is finitely generated as a profinite group, it is finite [53, Theorem 4.3.5].

Lemma 3.2. Let $\underline{G}$ be a linear algebraic $\mathbb{Q}$-group, and let $\Gamma \leq \underline{G}(\mathbb{Q})$ be an arithmetic subgroup. If $\Gamma$ has no finitely generated infinite normal solvable subgroup, then $\underline{G}$ is reductive and $\Gamma \cap \mathcal{D}(\underline{G})(\mathbb{Q})$ has finite index in $\Gamma$, where $\mathcal{D}(\underline{G})$ denotes the derived subgroup.

Proof. Upon passing to finite index subgroups of $\underline{G}$ and $\Gamma$, we may assume $\underline{G}$ is connected, so that due to [8, Théorème 7.15] and [44, Section 0.24], we have a decomposition

$$
\underline{G}=R_{u}(\underline{G}) \rtimes \underline{S} \mathcal{D}(\underline{G})
$$

as semidirect product of the unipotent radical $R_{u}(\underline{G})$ and a reductive $\mathbb{Q}$-subgroup $\underline{S} \mathcal{D}(\underline{G})$. The latter group is an almost direct product of the central $\mathbb{Q}$-torus $\underline{S}$ and the semisimple derived subgroup $\mathcal{D}(\underline{G})$.

By [8, Corollaire 7.13.(4)], $\Gamma$ is commensurable with the group $\Lambda=R_{u}(\underline{G})(\mathbb{Z})(\underline{S} \mathcal{D}(\underline{G}))(\mathbb{Z})$. Using [44, Corollary (3.2.9)], $\underline{S}(\mathbb{Z}) \mathcal{D}(\underline{G})(\mathbb{Z})$ and $(\underline{S} \mathcal{D}(\underline{G}))(\mathbb{Z})$ are commensurable. As arithmetic sub- 
groups of unipotent groups are Zariski dense [44, Lemma 3.3.3.(iii)], it follows that $R_{u}(\underline{G})(\mathbb{Z})$ is an infinite normal nilpotent subgroup of $\Lambda$ whenever $R_{u}(\underline{G})$ is not trivial. Moreover, arithmetic groups are finitely generated. So our assumption on $\Gamma$ implies that $R_{u}(\underline{G})$ is trivial and $\underline{G}$ is reductive. Since the group $\underline{S}(\mathbb{Z})$ is abelian and normal in $\Lambda$, which is commensurable to $\Gamma$, it has to be finite. Thus $\Gamma \cap \mathcal{D}(\underline{G})(\mathbb{Q})$ is of finite index in $\Gamma$.

One says that a profinite group is adelic if it is isomorphic to a closed subgroup of some $\mathrm{SL}_{m}(\widehat{\mathbb{Z}})$. See [41] for a discussion of this notion. One easily sees that a profinite group $G$ that contains an adelic subgroup $H<S L_{m}(\widehat{\mathbb{Z}})$ of finite index is itself adelic-via an embedding into $\operatorname{SL}_{m[G: H]}(\widehat{\mathbb{Z}})$.

Theorem 3.3 (Platonov-Rapinchuk, Lubotzky). Let $\underline{G}$ be a simply connected semisimple linear algebraic $\mathbb{Q}$-group such that each $\mathbb{Q}$-simple factor of $\underline{G}$ satisfies the Platonov-Margulis conjecture. Let $\Lambda<\underline{G}(\mathbb{Q})$ be an $S$-arithmetic subgroup. If $\widehat{\Lambda}$ is adelic, then $\underline{G}$ has a finite congruence kernel.

Proof. Being simply connected, the group $\underline{G}$ is a product of its $\mathbb{Q}$-simple factors $\underline{G}_{i}$; hence $\Lambda$ is commensurable with a product of arithmetic subgroups $\Lambda_{i} \subseteq \underline{G}_{i}(\mathbb{Q})$. If each $\underline{G}_{i}$ has a finite congruence kernel, then so has $\underline{G}$. Further, $\widehat{\Lambda}$ is adelic if and only if each $\widehat{\Lambda_{i}}$ is adelic. Hence we may and will assume that $\underline{G}$ is $\mathbb{Q}$-simple.

Since $\widehat{\Lambda}$ is an adelic group, it is boundedly generated by [41, Theorem 12.2]. Finally, by [41, Theorem 12.10], which depends on the Platonov-Margulis conjecture as a global assumption, $\underline{G}$ has a finite congruence kernel. To be more precise, the assumption in [41, Theorem 12.10] is that $\underline{G}$ is absolutely simple over a number field. But as a $\mathbb{Q}$-simple group, $\underline{G}$ is the Weil restriction of an absolutely simple group $\underline{H}$ over a number field. So by [41, Theorem 12.10], $\underline{H}$ has a finite congruence kernel, and hence $\underline{G}$ has a finite congruence kernel; see the remark at the beginning of Section 2.

Proof of Theorems 1.1 and 1.6 (i). By passing to finite index subgroups, we may assume that $\Gamma_{1}$ and $\Gamma_{2}$ are profinitely isomorphic. As in the proof before, we conclude from the congruence subgroup property of $\underline{G}_{1}$ that $\widehat{\Gamma}_{1} \cong \widehat{\Gamma}_{2}$ is adelic.

Assume first that $\Gamma_{1}$ has a finitely generated infinite normal solvable subgroup. Its closure is a (topologically) finitely generated infinite closed normal solvable subgroup of $\widehat{\Gamma}_{1} \cong \widehat{\Gamma}_{2}$. The $\ell^{2}$-Betti numbers of $\Gamma_{1}$ vanish by a result of Cheeger and Gromov [16], and thus $\chi\left(\Gamma_{1}\right)=0$.

If $\Gamma_{2}$ had an infinite normal solvable subgroup, then $\chi\left(\Gamma_{2}\right)=0$ for the same reason, and the proof would be finished. Otherwise, Lemma 3.2 would imply that $\underline{G}_{2}$ is reductive and, upon passing to finite index subgroups, $\Gamma_{2}$ is an arithmetic subgroup of the semisimple group $\mathcal{D}\left(\underline{G}_{2}\right)$. We show that this cannot happen, thus concluding the proof in the case that $\Gamma_{1}$ has a finitely generated infinite normal solvable subgroup. The preimage $\Lambda$ of $\Gamma_{2}$ in $\widetilde{\mathcal{D}\left(\underline{G}_{2}\right)}$ is commensurable with $\Gamma_{2}$ by [44, Corollary 3.2.9]. Hence $\widehat{\Lambda}$ is adelic because $\widehat{\Gamma}_{1} \cong \widehat{\Gamma}_{2}$ is. So $\widehat{\mathcal{D}\left(\underline{G}_{2}\right)}$ has a finite congruence kernel by Theorem 3.3 and the assumption regarding the Platonov-Margulis conjecture in Theorem 1.6. Moreover, $\widehat{\Lambda}$ contains (topologically) a finitely generated infinite closed normal solvable subgroup because $\widehat{\Gamma}_{1} \cong \widehat{\Gamma}_{2}$ does. According to Lemma 3.1, this is absurd.

Next we assume that $\Gamma_{1}$ has no finitely generated closed normal infinite solvable subgroup. By Lemma 3.2, the group $G_{1}$ is reductive. Its derived subgroup $\mathcal{D}\left(\underline{G}_{1}\right)$ has a finite congruence kernel as well [50, Lemma 2]. Again, by Lemma 3.2 and upon passing to a finite index subgroup of $\Gamma_{1}$, we may assume that $\underline{G}_{1}$ is semisimple and has a finite congruence kernel. By the argument at the beginning of the proof of Lemma 3.1, we may assume that $\underline{G}_{1}$ is simply connected. Hence $\underline{G}_{1}$ has strong approximation [49, Theorem 7.12].

By Lemma 3.1, the group $\widehat{\Gamma}_{1} \cong \widehat{\Gamma}_{2}$ has no (topologically) finitely generated infinite closed normal solvable subgroup. In particular, $\Gamma_{2}$ has no finitely generated infinite normal solvable subgroup. By Lemma 3.2, the group $\underline{G}_{2}$ is reductive, and, upon passing to finite index subgroups and replacing $\underline{G}_{2}$ by its derived subgroup, we may assume that $\Gamma_{2}$ is an $S$-arithmetic subgroup of the semisimple group $\underline{G}_{2}$. By passing to finite index subgroups once more and appealing to [44, Corollary 3.2.9] and replacing 
$\underline{G}_{2}$ by its simply connected covering, we may assume that $\Gamma_{2}$ is an $S$-arithmetic subgroup of the simply connected semisimple group $\underline{G}_{2}$, which satisfies strong approximation by [49, Theorem 7.12]. Since $\widehat{\Gamma}_{2}$ is adelic, $\underline{G}_{2}$ has a finite congruence kernel by Theorem 3.3.

We can then apply Proposition 2.4 to conclude that $S$ contains no finite places and Theorem 2.1 to obtain that $\operatorname{dim} X_{1}=\operatorname{dim} X_{2} \bmod 4$ and $\delta\left(G_{1}\right)=\delta\left(G_{2}\right)$. Note that for the proof of Theorem 1.1, we could just start at this point of the argument.

The semisimple Lie groups $G_{i}$ possess uniform lattices $\Lambda_{i} \leq G_{i}$, and $\Gamma_{i}$ is measure equivalent to $\Lambda_{i}$; see [22, Definition 2.1 and Example 2.2]. Gaboriau's proportionality principle [23, Théorème 6.3] implies that $b_{n}^{(2)}\left(\Gamma_{i}\right)=0$ if and only if $b_{n}^{(2)}\left(\Lambda_{i}\right)=0$. Borel [9] computed that $b_{n}^{(2)}\left(\Lambda_{i}\right) \neq 0$ if and only if $\delta\left(G_{i}\right)=0$ and $\operatorname{dim} X_{i}=2 n$. As we have $\chi\left(\Gamma_{i}\right)=\sum_{n \geq 0}(-1)^{n} b_{n}^{(2)}\left(\Gamma_{i}\right)$, it follows that

$$
\operatorname{sign} \chi\left(\Gamma_{i}\right)= \begin{cases}0 & \text { if } \delta\left(G_{i}\right)>0 \\ (-1)^{\operatorname{dim}\left(X_{i}\right) / 2} & \text { if } \delta\left(G_{i}\right)=0 .\end{cases}
$$

This formula can also be deduced using Harder's Gauß-Bonnet theorem [25] and Hirzebruch's proportionality principle. Be aware that $\delta\left(G_{i}\right)=0$ implies that $\operatorname{dim} X_{i}$ is even: since every root system has an even number of roots, it follows that $\delta\left(G_{i}\right)$ and $\operatorname{dim} X_{i}$ have the same parity. This completes the proof.

The proof of the profiniteness of $\operatorname{sign} \rho^{(2)}(\Gamma)$ is mostly parallel to the proof of profiniteness of $\operatorname{sign} \chi(\Gamma)$.

Proof of Theorem 1.4 and 1.6 (ii). In addition to having vanishing $\ell^{2}$-cohomology, groups of type $(F)$ with infinite elementary amenable normal subgroups also have vanishing $\ell^{2}$-torsion [42, Theorem 3.113]. Hence, as in the previous proof, we may assume that $\underline{G}_{1}$ and $\underline{G}_{2}$ are semisimple and $S$ contains no finite places. Further, we obtain $\operatorname{dim} X_{1}=\operatorname{dim} X_{2} \bmod 4$ and $\delta\left(G_{1}\right)=\delta\left(G_{2}\right)$. Since $\operatorname{rk}_{\mathbb{Q}} \underline{G}_{1}=\operatorname{rk}_{\mathbb{Q}} \underline{G}_{2}=$ 0 , the arithmetic subgroups $\Gamma_{i}$ are uniform lattices in $G_{i}$. Thus, using the equality of topological and analytic $\ell^{2}$-torsion for closed manifolds [14], a result of Olbrich [48, Theorem 1.1.(c)] gives $\rho^{(2)}\left(\Gamma_{i}\right) \neq 0$ if and only if $\delta\left(G_{i}\right)=1$. From Olbrich's formulas in [48, Proposition 1.3], it follows moreover that if $\delta\left(G_{i}\right)=1$, then $\operatorname{sign} \rho^{(2)}\left(\Gamma_{i}\right)=(-1)^{\left(\operatorname{dim} X_{i}-1\right) / 2}$. This completes the proof of Theorem 1.4.

\section{The Euler characteristic of arithmetic spin groups}

In this final section, we explicitly compute the Euler characteristic of arithmetic spin groups; and, as a particular case, we obtain the proof of Theorem 1.2.

Let $V$ be a free $\mathbb{Z}$-module of finite rank $d$ with a symmetric bilinear form $b: V \times V \rightarrow \mathbb{Z}$. We will assume that the form $b$ is non-singular: that is, for every primitive vector $v \in V$, there is some $w \in V$ with $b(v, w)=1$.

The following examples will be of interest for us. Let $m, n \geq 0$ be integers, and define $d=m+n$. We consider $V_{m, n}=\mathbb{Z}^{d}$ with the standard basis $e_{1}, \ldots, e_{d}$. The bilinear form $b_{m, n}$ defined by

$$
b_{m, n}\left(e_{i}, e_{j}\right)= \begin{cases}1 & \text { if } i=j \leq m \\ -1 & \text { if } i=j>m \\ 0 & \text { if } i \neq j\end{cases}
$$

is non-singular.

For every commutative ring $A$, we put $V_{A}=A \otimes_{\mathbb{Z}} V$, and we write $b_{A}$ for the $A$-bilinear extension of $b$. We get an associated Clifford algebra

$$
C\left(V_{A}, b_{A}\right)=T_{A}\left(V_{A}\right) /\left(v^{2}-b_{A}(v, v)\right)
$$


as a quotient of the tensor algebra $T_{A}\left(V_{A}\right)$ of $V_{A}$. As $A$-module the Clifford algebra is free and, if $e_{1}, \ldots, e_{d}$ is a basis of $V$, then a basis of $C\left(V_{A}, b_{A}\right)$ is given by the elements

$$
e(J)=e_{j_{1}} \cdot e_{j_{2}} \cdots e_{j_{s}}
$$

for every subset $J=\left\{j_{1}, j_{2}, \ldots, j_{s}\right\} \subseteq\{1, \ldots, d\}$ with $j_{1}<j_{2}<\cdots<j_{s}$; see [38, IV (1.5.1)]. Here, the convention $e(\emptyset)=1$ is used. As a consequence, $A \otimes_{\mathbb{Z}} C(V, b) \cong C\left(V_{A}, b_{A}\right)$. The Clifford algebra is $\mathbb{Z} / 2 \mathbb{Z}$ graded and decomposes as $C\left(V_{A}, b_{A}\right)=C_{0}\left(V_{A}, b_{A}\right) \oplus C_{1}\left(V_{A}, b_{A}\right)$, where $C_{0}\left(V_{A}, b_{A}\right)$ is spanned by the $e(J)$ for sets $J$ of even cardinality.

We note that there is a unique anti-automorphism $\iota: C\left(V_{A}, b_{A}\right) \rightarrow C\left(V_{A}, b_{A}\right)$ with $\iota(v)=v$ for all $v \in V_{A}$ (of order two). Moreover, the grading yields an involution $x \mapsto x^{\prime}$ with $x=x_{0}+x_{1}$ and $x^{\prime}=x_{0}-x_{1}$ for all $x_{0} \in C_{0}\left(V_{A}, b_{A}\right)$ and $x_{1} \in C_{1}\left(V_{A}, b_{A}\right)$. Composition of these two maps yields the conjugation $x \mapsto \bar{x}=\iota\left(x^{\prime}\right)=\iota(x)^{\prime}$ on the Clifford algebra.

Definition 4.1. For a commutative ring $A$, the spin group of $b$ over $A$ is defined by

$$
\underline{\operatorname{Spin}}(b)(A)=\left\{g \in C_{0}\left(V_{A}, b_{A}\right) \mid g \bar{g}=1 \text { and } g V_{A} \bar{g}=V_{A}\right\} .
$$

The functor $\operatorname{Spin}(b)$ from the category of commutative rings to the category of groups is an affine group scheme of finite type over $\mathbb{Z}$. In the following, we investigate only spin groups for the forms $b=b_{m, n}$. In this case, the basis vectors satisfy $e_{i} \cdot e_{j}=-e_{j} \cdot e_{i}$ in the Clifford algebra for all $i \neq j$. Hence, we have $\iota(e(J))=(-1)^{|J|(|J|-1) / 2} e(J)$, and therefore the identity

$$
\overline{e(J)}=(-1)^{|J|(|J|+1) / 2} e(J)
$$

holds for every subset $J \subseteq\{1, \ldots, m+n\}$.

Definition 4.2. Let $m, n>0$ be integers. In $\operatorname{Spin}\left(b_{m, n}\right)(\mathbb{Z})$, we define the principal congruence subgroup of level 4 as

$$
\Gamma_{m, n}=\operatorname{ker}\left(\underline{\operatorname{Spin}}\left(b_{m, n}\right)(\mathbb{Z}) \rightarrow \underline{\operatorname{Spin}}\left(b_{m, n}\right)(\mathbb{Z} / 4 \mathbb{Z})\right) .
$$

Remark 4.3. We decided to work with the principal congruence subgroup of level 4 for two reasons. First, a classical result of Minkowski shows that the principal congruence group $\Gamma_{m, n}$ is torsion-free (see [33, III.2.3] for a formulation in terms of group schemes). Therefore, the work of Borel-Serre implies that the arithmetic group $\Gamma_{m, n}$ is a group of type $F[10,11.1]$.

The second reason is that, as we shall see below, the group scheme $\operatorname{Spin}\left(b_{m, n}\right)$ is not smooth at the prime 2. Passing to the congruence subgroup of level 4 avoids some technicalities in the computation of the Euler characteristic.

Theorem 4.4. Let $d \geq 3$ with $d=m+n$ for integers $m, n \geq 1$. Put $\ell=\left\lfloor\frac{d}{2}\right\rfloor$ and $k=\left\lfloor\frac{m}{2}\right\rfloor$. If $m$ and $n$ are odd, then $\chi\left(\Gamma_{m, n}\right)=0$.

If at least one of $m$ and $n$ is even, then

$$
\chi\left(\Gamma_{m, n}\right)=(-1)^{m n / 2} R(d)\left(\begin{array}{l}
\ell \\
k
\end{array}\right) \prod_{j=1}^{\ell-1}\left(\left(2^{2 j}-1\right)|\zeta(1-2 j)|\right),
$$

where $R(d)$ is

$$
R(d)= \begin{cases}2^{5 \ell^{2}-4 \ell}\left(2^{\ell}-1\right)|\zeta(1-\ell)| & \text { if } d \equiv 0 \bmod 4 \\ 2^{5 \ell^{2}-5 \ell+1} \frac{\left|B_{\psi, \ell}\right|}{\ell} & \text { if } d \equiv 2 \bmod 4 \\ 2^{5 \ell^{2}}\left(2^{d-1}-1\right)|\zeta(2-d)| & \text { if } d \equiv 1 \bmod 2 .\end{cases}
$$

Here, $B_{\psi, \ell}$ is the $\ell$-th generalized Bernoulli number with respect to the primitive Dirichlet character $\psi$ modulo 4 . 
Remark 4.5. For the definition of the generalized Bernoulli numbers, we refer to [47]. We have $B_{\psi, \ell}=0$ exactly if $\ell$ is even, a case that does not occur in the formula. The generalized Bernoulli numbers can be computed easily. For convenience, we list the first values:

\begin{tabular}{l|c|c|c|c|c}
$\ell$ & 1 & 3 & 5 & 7 & 9 \\
\hline$B_{\psi, \ell}$ & $-\frac{1}{2}$ & $\frac{3}{2}$ & $-\frac{25}{2}$ & $\frac{427}{2}$ & $-\frac{12465}{2}$
\end{tabular}

Assuming Theorem 4.4 for the moment, we obtain the proof of Theorem 1.2 as a special case.

Proof of Theorem 1.2. We consider the groups $\Gamma_{8,2} \subseteq \operatorname{Spin}(8,2)$ and $\Gamma_{4,6} \subseteq \operatorname{Spin}(4,6)$. It follows from [3] that these groups are profinitely isomorphic. We briefly recall the argument. The algebraic groups $\operatorname{Spin}\left(b_{8,2}\right) \times_{\mathbb{Z}} \mathbb{Q}$ and $\operatorname{Spin}\left(b_{4,6}\right) \times_{\mathbb{Z}} \mathbb{Q}$ are simple and simply connected, and the associated real Lie groups $\operatorname{Spin}(8,2)=\operatorname{Spin}\left(b_{8,2}\right)(\mathbb{R})$ and $\operatorname{Spin}(4,6)=\operatorname{Spin}\left(b_{4,6}\right)(\mathbb{R})$ are not compact. Hence, both algebraic groups have strong approximation; see [49, Theorem 7.2]. Moreover, the quadratic forms $b_{8,2}$ and $b_{4,6}$ have Witt index 2 and 4 , respectively; therefore, according to $[37,11.3]$, the congruence kernels of $\underline{\operatorname{Spin}}\left(b_{8,2}\right)$ and $\underline{\operatorname{Spin}}\left(b_{4,6}\right)$ are trivial. We deduce that

$$
\widehat{\Gamma}_{8,2}=K_{8,2} \times \prod_{p \text { odd }} \underline{\operatorname{Spin}}\left(b_{8,2}\right)\left(\mathbb{Z}_{p}\right)
$$

and

$$
\widehat{\Gamma}_{4,6}=K_{4,6} \times \prod_{p \text { odd }} \underline{\operatorname{Spin}}\left(b_{4,6}\right)\left(\mathbb{Z}_{p}\right),
$$

where $K_{m, n}=\operatorname{ker}\left(\operatorname{Spin}\left(b_{m, n}\right)\left(\mathbb{Z}_{2}\right) \rightarrow \operatorname{Spin}\left(b_{m, n}\right)(\mathbb{Z} / 4 \mathbb{Z})\right)$ is the open compact principal congruence subgroup of level 4 . However, the forms $\overline{b_{8,2}}$ and $b_{4,6}$ are isometric over $\mathbb{Z}_{p}$ for every prime number $p$; see [3, Corollary 3]. Thus the group schemes $\operatorname{Spin}\left(b_{8,2}\right) \times_{\mathbb{Z}} \mathbb{Z}_{p}$ and $\operatorname{Spin}\left(b_{4,6}\right) \times_{\mathbb{Z}} \mathbb{Z}_{p}$ are isomorphic for every prime $p$. In particular, $\operatorname{Spin}\left(b_{8,2}\right)\left(\mathbb{Z}_{p}\right) \cong \operatorname{Spin}\left(b_{4,6}\right)\left(\mathbb{Z}_{p}\right)$ and $K_{8,2} \cong K_{4,6}$; we deduce that the profinite completions are isomorphic.

Now we use Theorem 4.4 to compute the Euler characteristic. We have $d=10$ and $\ell=5$, and thus we obtain $R(d)=2^{100} \cdot 5$. Since $\zeta(-1)=-1 /(12), \zeta(-3)=1 /(120), \zeta(-5)=-1 /(252)$, and $\zeta(-7)=1 /(240)$ (see also [20, Section 1.5]), the product evaluates as

$$
\prod_{j=1}^{4}\left(\left(2^{2 j}-1\right)|\zeta(1-2 j)|\right)=\frac{3 \cdot 15 \cdot 63 \cdot 255}{12 \cdot 120 \cdot 252 \cdot 240}=\frac{17}{2^{11}}
$$

For $m=8$, we have $k=4$; and with $\left(\begin{array}{l}5 \\ 4\end{array}\right)=5$, we obtain

$$
\chi\left(\Gamma_{8,2}\right)=2^{89} \cdot 5^{2} \cdot 17
$$

For $m=4$, we have $k=2$; and since $\left(\begin{array}{l}5 \\ 2\end{array}\right)=10$, we have

$$
\chi\left(\Gamma_{4,6}\right)=2^{90} \cdot 5^{2} \cdot 17
$$

To prove Theorem 4.4, we need some preparation. For simplicity, from now on we will write $b$ for $b_{m, n}$, and we set $\underline{G}=\operatorname{Spin}\left(b_{m, n}\right)$. As a first step, we determine the Lie algebra of $\underline{G}$. It follows from the next lemma that the group scheme $\underline{G} \times_{\mathbb{Z}} \mathbb{Z}_{p}$ is smooth if $p \neq 2$ but is not smooth for $p=2$. This problem forces us to be more careful when dealing with the prime $p=2$. 
Lemma 4.6. Let $A$ be a commutative ring, and let $\operatorname{ann}(2 A)=\{a \in A \mid 2 a=0\}$ be the annihilator of $2 A$. The Lie algebra of $\underline{G}$ over $A$ is isomorphic to the Lie subalgebra of $C_{0}\left(V_{A}, b_{A}\right)$ given by

$$
\operatorname{Lie}(\underline{G})(A) \cong \bigoplus_{|J|=2} A e(J) \oplus \bigoplus_{\substack{\text { Jeven } \\|J| \neq 2}} \operatorname{ann}(2 A) e(J),
$$

where the sums run over subsets $J \subseteq\{1, \ldots, d\}$ of even cardinality.

Proof. Consider the ring $A[\varepsilon]$ with $\varepsilon^{2}=0$. Recall that the Lie algebra is defined as

$$
\operatorname{Lie}(\underline{G})(A)=\left\{X \in C_{0}\left(V_{A}, b_{A}\right) \mid 1+\varepsilon X \in \underline{G}(A[\varepsilon])\right\} .
$$

Let $X \in C_{0}\left(V_{A}, b_{A}\right)$. Write

$$
X=\sum_{J \text { even }} x_{J} e(J)
$$

with coefficients $x_{J} \in A$, and define $g=1+\varepsilon X$. Note that $g^{-1}=1-\varepsilon X$. We determine under which conditions $X \in \operatorname{Lie}(\underline{G})(A)$.

Using (4.1), we see that $1=g \bar{g}=1+\varepsilon(X+\bar{X})$ holds exactly if $x_{J} \in \operatorname{ann}(2 A)$ for all $J$ with $|J| \equiv 0 \bmod 4$. Moreover, $g$ satisfies $g e_{i} g^{-1} \in V_{A[\varepsilon]}$ if and only if $X e_{i}-e_{i} X \in V_{A}$ for all $i$. Let $J \subseteq\{1, \ldots, d\}$ be a set with an even number of elements. Then $e(J) e_{i}-e_{i} e(J)=0$ if $i \notin J$. However, if $i \in J$, then

$$
e(J) e_{i}-e_{i} e(J)=e_{i}^{2}(-1)^{|\{j \in J \mid j>i\}|} 2 e(J \backslash\{i\}) .
$$

We deduce that $X e_{i}-e_{i} X \in V_{A}$ is satisfied precisely when $x_{J} \in \operatorname{ann}(2 A)$ for all $J$ with $|J|>2$. We leave it to the reader to verify that the Lie algebra structure is indeed induced by the commutator bracket on $C_{0}\left(V_{A}, b_{A}\right)$. For instance, one can use the formula given in [17, II, Section 4, 4.2].

Proof of Theorem 4.4. We first fix some notation. Let $\underline{G}(\mathbb{R})=\operatorname{Spin}(m, n)$ be the associated real spin group. The Lie algebra $\mathcal{L}(\operatorname{Spin}(m, n))$ will be denoted by $\mathfrak{g}$. We identify $\mathfrak{g}$ with a Lie subalgebra of the Clifford algebra $C\left(V_{\mathbb{R}}, b_{\mathbb{R}}\right)$; see also Lemma 4.6. The vectors $e(J)$ where $J$ runs through the two-element subsets of $\{1, \ldots, d\}$ are a basis of $\mathfrak{g}$. The subalgebra $\mathfrak{f}$ spanned by the $e(J)$ where $J \subseteq\{1, \ldots, m\}$ or $J \subseteq\{m+1, \ldots, m+n\}$ is maximal compact. The corresponding maximal compact subgroup will be denoted by $K_{\infty}$. A Cartan decomposition is given by $\mathfrak{g}=\mathfrak{f} \oplus \mathfrak{p}$, where $\mathfrak{p}$ is spanned by the $e(J)$ with $J=\{i, j\}$ satisfying $i \leq m<j$.

Let $X=\operatorname{Spin}(m, n) / K_{\infty}$ denote the associated Riemannian symmetric space. Note that $\operatorname{dim}(X)=m n$ and $\operatorname{dim}\left(\underline{G} \times_{\mathbb{Z}} \mathbb{Q}\right)=d(d-1) / 2$, where $d=m+n$. Since $X$ is simply connected and $\Gamma_{m, n}$ acts freely and properly on $X$, the quotient space $X / \Gamma_{m, n}$ is the classifying space of $\Gamma_{m, n}$. We will calculate the Euler characteristic of this space. If $m$ and $n$ are odd, then the Euler-Poincaré measure on $\operatorname{Spin}(m, n)$ vanishes and $\chi\left(\Gamma_{m, n}\right)=0$; see [56] or [34, Theorem 3.1]. From now on, we assume that $\operatorname{dim}(X)=m n$ is even.

The linear algebraic group $\underline{G} \times_{\mathbb{Z}} \mathbb{Q}$ is simple and simply connected [45, Theorem 24.61]. The associated real Lie group $\underline{G}(\mathbb{R})=\operatorname{Spin}(m, n)$ is not compact, since we assume $m, n \geq 1$. We infer that $\underline{G}$ has strong approximation; see [49, Theorem 7.12]. It follows that the inclusion $\operatorname{Spin}(m, n) \rightarrow \underline{G}(\mathbb{A})$ induces a homeomorphism

$$
\underline{G}(\mathbb{Q}) \backslash \underline{G}(\mathbb{A}) / K_{\infty} K_{f} \cong X / \Gamma_{m, n}
$$

where $K_{f}=K_{m, n}^{(2)} \times \prod_{p \text { odd }} \underline{G}\left(\mathbb{Z}_{p}\right)$ is an open compact subgroup with

$$
K_{m, n}^{(2)}=\operatorname{ker}\left(\underline{G}\left(\mathbb{Z}_{2}\right) \rightarrow \underline{G}(\mathbb{Z} / 4 \mathbb{Z})\right) .
$$

We will compute the Euler characteristic using the adelic formula given in Theorem 3.3 in [34]. We choose $B$ to be the symmetric bilinear form on $\operatorname{Lie}(\underline{G})(\mathbb{Q})$ for which the vectors $e(J)$ are an orthonormal basis. The form $B$ is nice in the sense of [34]: that is, the Cartan decomposition on $\mathfrak{g}=\mathfrak{f} \oplus \mathfrak{p}$ given 
above is orthogonal. Moreover, $B$ induces a volume form $\operatorname{vol}_{B}$ on $\underline{G}\left(\mathbb{Q}_{v}\right)$ at every place of $v$ of $\mathbb{Q}$. Now Theorem 3.3 in [34] yields

$$
\chi\left(X / \Gamma_{m, n}\right)=(-1)^{m n / 2} \frac{\left|W\left(\mathfrak{g}_{\mathbb{C}}\right)\right| \tau(\underline{G})}{\left|W\left(\mathfrak{f}_{\mathbb{C}}\right)\right|} \operatorname{vol}_{B}\left(G_{u}\right)^{-1} \operatorname{vol}_{B}\left(K_{f}\right)^{-1},
$$

where $\tau(\underline{G})$ is the Tamagawa number of $\underline{G}$ and $W\left(\mathfrak{g}_{\mathbb{C}}\right)$ and $W\left(\mathfrak{f}_{\mathbb{C}}\right)$ denote the Weyl groups of the complexified Lie algebras of $\mathfrak{g}_{\mathbb{C}}$ and $\mathfrak{f}_{\mathbb{C}}$ respectively. Moreover, $G_{u}$ denotes the compact dual group: that is, the compact group $\operatorname{Spin}(d)$ in our case.

Now we evaluate the terms in the formula step by step. We put $\ell=\left\lfloor\frac{d}{2}\right\rfloor$ and $k=\left\lfloor\frac{m}{2}\right\rfloor$ and $k^{\prime}=\left\lfloor\frac{n}{2}\right\rfloor$. Observe that $\ell=k+k^{\prime}$ since we excluded the case that both $m$ and $n$ are odd.

In evaluating the volume $\operatorname{vol}_{B}\left(K_{f}\right)$ there is, however, a subtle point: the adelic formula in [34] is based on the assumption that the underlying group scheme is smooth over $\mathbb{Z}$. This assumption is only used in evaluating $\operatorname{vol}_{B}\left(K_{f}\right)$. As we have seen in 4.6, our group scheme $\underline{G}$ is not smooth over $\mathbb{Z}$ since there is a problem at the prime 2 . In the last step, we shall take care of this problem.

Tamagawa number: $\tau(\underline{G})=1$.

Since $d \geq 2$, the spin group $\underline{G} \times \mathbb{Z} \mathbb{Q}$ is semisimple and simply connected. The assertion follows from Kottwitz's Tamagawa number theorem [40]. For spin groups, this was already observed by Tamagawa and Weil.

Orders of Weyl groups: $\frac{\left|W\left(\mathfrak{g}_{\mathrm{C}}\right)\right|}{\left|W\left(\mathfrak{f}_{\mathrm{C}}\right)\right|}=2\left(\begin{array}{l}\ell \\ k\end{array}\right)$.

If $d=2 \ell$ is even, then $\mathfrak{g}_{\mathbb{C}}$ is a simple Lie algebra of type $D_{\ell}$, and $\mathfrak{f}_{\mathbb{C}}$ is a product of simple Lie algebras of type $D_{k}$ and $D_{k^{\prime}}$. The table in [27] yields

$$
\frac{\left|W\left(\mathfrak{g}_{\mathbb{C}}\right)\right|}{\left|W\left(\mathfrak{f}_{\mathbb{C}}\right)\right|}=\frac{2^{\ell-1} \ell !}{2^{k-1} k ! 2^{k^{\prime}-1} k^{\prime} !}=2\left(\begin{array}{l}
\ell \\
k
\end{array}\right)
$$

Similarly, if $d=2 \ell+1$, then $\mathfrak{g}_{\mathbb{C}}$ is a simple Lie algebra of type $B_{\ell}$, and the Weyl group has order $2^{\ell} \ell$; see [27]. Now $\mathfrak{f}_{\mathbb{C}}$ is a product of two simple Lie algebras either of types $B_{k}$ and $D_{k^{\prime}}$ or of types $D_{k}$ and $B_{k^{\prime}}$. A short calculation yields the formula.

Volume of $G_{u}: \operatorname{vol}_{B}\left(G_{u}\right)=2^{\left(3 d-d^{2}\right) / 2} \prod_{j=2}^{d} \pi^{j / 2} \Gamma(j / 2)^{-1}$.

The compact dual group $G_{u}$ is $\operatorname{Spin}(d)$. Since $\operatorname{Spin}(d)$ is a twofold covering of $\operatorname{SO}(d)$, we obtain $\operatorname{vol}_{B}(\operatorname{Spin}(d))=2 \operatorname{vol}_{B}(\mathrm{SO}(d))$. However, we have to relate the induced left-invariant Riemann metric $B$ to the standard left-invariant metric $\gamma$ on $\mathrm{SO}(d)$. More precisely, the vectors $v_{i, j}=E_{i, j}-E_{j, i}$ with $i<j \leq d$, where $E_{i, j}$ denotes the elementary matrix with entry 1 in position $(i, j)$, form a basis of the Lie algebra $\mathfrak{s} \mathfrak{p}(d)$. At the identity $\gamma$ is the symmetric bilinear form for which $\left(v_{i, j}\right)_{i<j}$ is an orthonormal basis. Using induction one shows that the volume with respect to $\gamma$ is

$$
\operatorname{vol}_{\gamma}(\mathrm{SO}(d))=\prod_{j=2}^{d} \operatorname{vol}\left(S^{j-1}\right)=\prod_{j=2}^{d} 2 \frac{\pi^{j / 2}}{\Gamma(j / 2)} .
$$

The tangent map of the projection $p: \operatorname{Spin}(d) \rightarrow \mathrm{SO}(d)$ maps the basis vector $e(I)$ with $I=\{i, j\}$ and $i<j$ to $2 v_{i, j}$; therefore, $B=\frac{1}{4} \gamma$ and

$$
\operatorname{vol}_{B}(\mathrm{SO}(d))=2^{-d(d-1) / 2} \operatorname{vol}_{\gamma}(\mathrm{SO}(d)) .
$$

Local volume $\operatorname{vol}_{B}\left(K_{f}\right)$ :

Here we obtain a formula for the local volume

$$
\operatorname{vol}_{B}\left(K_{f}\right)=\operatorname{vol}_{B}\left(K_{m, n}^{(2)}\right) \prod_{p \text { odd }} \operatorname{vol}_{B}\left(\underline{G}\left(\mathbb{Z}_{p}\right)\right) .
$$


As in [34], we use the smoothness of $\underline{G} \times_{\mathbb{Z}} \mathbb{Z}_{p}$ for all odd primes to apply Weil's formula $\operatorname{vol}_{B}\left(\underline{G}\left(\mathbb{Z}_{p}\right)\right)=$ $\left|\underline{G}\left(\mathbb{F}_{p}\right)\right| p^{-\operatorname{dim} \underline{G}}$. The special $p=2$ is discussed in Lemma 4.7 below, which yields $\operatorname{vol}_{B}\left(K_{m, n}^{(2)}\right)=$ $4^{-d}(d-1) / 2=2^{-d(d-1)}$. It remains to evaluate the infinite product over all odd primes

$$
\left.\prod_{p \text { odd }} \underline{G}\left(\mathbb{F}_{p}\right)\right|^{-1} p^{d(d-1) / 2} .
$$

Recall that over $\mathbb{F}_{p}$, there are exactly two quadratic forms in $d$ variables. They are uniquely determined by their discriminant in $\mathbb{F}_{p}^{\times} /\left(\mathbb{F}_{p}^{\times}\right)^{2}$; see [55, Theorem 3.8]. We note that the canonical map $\underline{G}\left(\mathbb{F}_{p}\right) \rightarrow$ $\underline{\mathrm{SO}}(m, n)\left(\mathbb{F}_{p}\right)$ has a two-element kernel; and, as $d \geq 3$, the image has index 2 in $\underline{\mathrm{SO}}(m, n)\left(\mathbb{F}_{p}\right)$, and thus $\left|\underline{G}\left(\mathbb{F}_{p}\right)\right|=\left|\underline{\operatorname{SO}}(m, n)\left(\mathbb{F}_{p}\right)\right|$.

Case 1: $d=2 \ell$ is even.

Let $p$ be an odd prime number. By assumption, $m$ and $n$ are even, and hence the $\operatorname{discriminant} \operatorname{det}\left(b_{m, n}\right)=$ 1. If a quadratic space $(V, q)$ of dimension $d$ over $\mathbb{F}_{p}$ splits as an orthogonal sum of hyperbolic planes, then we say that $q$ is of $\oplus$-type. Otherwise, $q$ has an anisotropic kernel of dimension 2 , and we say that $q$ is of $\ominus$-type.

Case 1a: $d \equiv 0 \bmod 4$.

In this case, $\ell$ is even, and thus $\operatorname{det}\left(b_{m, n}\right)=1=(-1)^{\ell}=\operatorname{det}\left(H^{\perp \ell}\right)$, where $H$ denotes the hyperbolic plane. We deduce that $b_{m, n}$ is of $\oplus$-type over $\mathbb{F}_{p}$. In this case, $\left|\underline{G}\left(\mathbb{F}_{p}\right)\right|=p^{\ell(\ell-1)}\left(p^{\ell}-1\right) \prod_{j=1}^{\ell-1}\left(p^{2 j}-1\right)$; see [4]. We obtain

$$
\begin{aligned}
\prod_{p \text { odd }}\left|\underline{G}\left(\mathbb{F}_{p}\right)\right|^{-1} p^{d(d-1) / 2} & =\prod_{p \text { odd }}\left(1-p^{-\ell}\right)^{-1} \prod_{j=1}^{\ell-1}\left(1-p^{-2 j}\right)^{-1} \\
& =\zeta(\ell)\left(1-2^{-\ell}\right) \prod_{j=1}^{\ell-1} \zeta(2 j)\left(1-2^{-2 j}\right),
\end{aligned}
$$

where $\zeta$ is the Riemann zeta function.

Case $1 \mathrm{~b}: d \equiv 2 \bmod 4$.

In this case, $\ell$ is odd; and, as in Case 1 a, we see that $b_{m, n}$ is of $\oplus$-type exactly if -1 is a square: that is, $p \equiv 1 \bmod 4$. Let $\psi$ denote the unique primitive Dirichlet character modulo 4 . Then the order of the spin group is $\left|\underline{G}\left(\mathbb{F}_{p}\right)\right|=p^{\ell(\ell-1)}\left(p^{\ell}-\psi(p)\right) \prod_{j=1}^{\ell-1}\left(p^{2 j}-1\right)$; see [4]. Using this, we obtain

$$
\begin{aligned}
\prod_{p \text { odd }}\left|\underline{G}\left(\mathbb{F}_{p}\right)\right|^{-1} p^{d(d-1) / 2} & =\prod_{p \text { odd }}\left(1-\psi(p) p^{-\ell}\right)^{-1} \prod_{j=1}^{\ell-1}\left(1-p^{-2 j}\right)^{-1} \\
& =L(\psi, \ell) \prod_{j=1}^{\ell-1} \zeta(2 j)\left(1-2^{-2 j}\right),
\end{aligned}
$$

where $L(\psi, s)$ is the Dirichlet $L$-function attached to $\psi$.

Case 2: $d=2 \ell+1$ is odd.

In this case, the order is $\left|\underline{G}\left(\mathbb{F}_{p}\right)\right|=p^{\ell^{2}} \prod_{j=1}^{\ell}\left(p^{2 j}-1\right)$; see [4]. Consequently, we obtain the formula

$$
\prod_{p \text { odd }}\left|\underline{G}\left(\mathbb{F}_{p}\right)\right|^{-1} p^{d(d-1) / 2}=\prod_{j=1}^{\ell} \zeta(2 j)\left(1-2^{-2 j}\right) .
$$

Finally, we multiply the terms and simplify using the functional equations of the Riemann zeta function and the Dirichlet $L$-function. More precisely, the functional equation of the $\zeta$-function [47, 
VII. (1.6)] and the well-known identity $\Gamma\left(\frac{1}{2}-j\right) \Gamma\left(\frac{1}{2}+j\right)=(-1)^{j} \pi$ imply that

$$
\zeta(2 j) \pi^{-2 j / 2} \Gamma\left(\frac{2 j}{2}\right) \pi^{-(2 j+1) / 2} \Gamma\left(\frac{2 j+1}{2}\right)=|\zeta(1-2 j)| .
$$

This identity makes it possible to combine one factor of the product in $\operatorname{vol}_{B}\left(K_{f}\right)^{-1}$ with two consecutive factors of the product occurring in $\operatorname{vol}_{B}\left(G_{u}\right)^{-1}$. If $d$ is even, there is a remaining term that needs to be simplified. If $d \equiv 0 \bmod 4$, then $\zeta(\ell) \pi^{-\ell} \Gamma(\ell)=2^{\ell-1}|\zeta(1-\ell)|$, as can be seen using the functional equation. For the case $d \equiv 2 \bmod 4$, the functional equation of the $L$-function [47, VII. (2.8)] yields

$$
L(\psi, \ell) \pi^{-\ell} \Gamma(\ell)=2^{-\ell}|L(\psi, 1-\ell)| .
$$

Eventually, we use $L(\psi, 1-\ell)=-\frac{B_{\psi, \ell}}{\ell}$ to express the special $L$-value in terms of generalized Bernoulli numbers; see [47, VII. (2.9)].

Lemma 4.7. In the notation above, we have $\operatorname{vol}_{B}\left(K_{m, n}^{(2)}\right)=4^{-d(d-1) / 2}$.

Proof. We construct an explicit chart that will allow us to compute the volume. The exponential series converges on $4 C_{0}\left(V_{\mathbb{Z}_{2}}, b\right)$ and defines an analytic function with values in $1+4 C_{0}\left(V_{\mathbb{Z}_{2}}, b\right)$. Let $x \in 4 \operatorname{Lie}(\underline{G})\left(\mathbb{Z}_{2}\right)$. Then $\bar{x}=-x$ commutes with $x$, and thus $\exp (x) \overline{\exp (x)}=\exp (x-x)=1$. Moreover, we claim that $\exp (x) v \overline{\exp (x)} \in V_{\mathbb{Z}_{2}}$ for every $v \in V_{\mathbb{Z}_{2}}$. Indeed, consider the endomorphism ad $\operatorname{ad}_{x}$ of $V_{\mathbb{Z}_{2}}$ defined by $\operatorname{ad}_{x}(v)=x v-v x$. Then a short calculation yields

$$
\exp (x) v \overline{\exp (x)}=\exp \left(\operatorname{ad}_{x}\right)(v) \in V_{\mathbb{Z}_{2}} .
$$

We deduce that the exponential function maps $4 \operatorname{Lie}(\underline{G})\left(\mathbb{Z}_{2}\right)$ to the group $K_{m, n}^{(2)}$. Similarly, the logarithmic series $L(1+a)=\sum_{k=1}^{\infty} \frac{(-1)^{k-1}}{k} a^{k}$ converges on $K_{m, n}^{(2)}$, and with similar arguments, one verifies that $L\left(K_{m, n}^{(2)}\right) \subseteq 4 \operatorname{Lie}(\underline{G})\left(\mathbb{Z}_{2}\right)$. Since $\exp$ and $L$ are inverses of each other, we deduce that

$$
\exp : 4 \operatorname{Lie}(\underline{G})\left(\mathbb{Z}_{2}\right) \rightarrow K_{m, n}^{(2)}
$$

is an analytic isomorphism. It is straightforward to check that the pullback of the volume density on $K_{m, n}^{(2)}$ via the exponential map to $4 \operatorname{Lie}(\underline{G})\left(\mathbb{Z}_{2}\right)$ yields the standard volume. As a consequence, we obtain $\operatorname{vol}_{B}\left(K_{m, n}^{(2)}\right)=4^{-\operatorname{dim}} \underline{G}$.

Remark 4.8. The factors that eventually distinguish the Euler characteristics of the profinitely isomorphic lattices $\Gamma_{m, n}$ and $\Gamma_{m-4, n+4}$ are the orders of the involved Weyl groups. These factors are special to the normalization of the Euler-Poincaré measure. However, it seems possible that profinitely isomorphic lattices in simple Lie groups have equal covolume with respect to some other geometrically relevant normalization of measures.

Example 4.9. As discussed in the introduction, our methods do not suffice to prove Theorem 1.1 for $S$-arithmetic groups. The sign of the Euler characteristic of an arithmetic group depends only on the Archimedean place, whereas for $S$-arithmetic groups, the sign depends on all places in $S$. This makes it necessary to understand the subtle interplay between the places. One class of examples that illustrates this behavior quite well are special linear groups over quaternion algebras. Other intriguing examples arise from $S$-arithmetic spin groups, as we will see now.

We consider the set $S=\{2, \infty\}$ of places of the field $\mathbb{Q}$ and the two groups $\operatorname{Spin}\left(b_{4,1}\right)$ and $\operatorname{Spin}\left(b_{2,3}\right)$. The quadratic forms $b_{4,1}$ and $b_{2,3}$ are equivalent over $\mathbb{Z}_{p}$ for every prime $p>\overline{2}$. Indeed, for odd primes, $b_{3,0}$ is isotropic over $\mathbb{Z}_{p}$; see [15, Lemma 1.7]. Hence, $b_{3,0}$ splits into a hyperbolic plane and a $b_{0,1}$. This proves the assertion since $b_{1,1}$ is equivalent to the hyperbolic plane over $\mathbb{Z}_{p}$. We note further that $b_{3,0}$ is anisotropic over $\mathbb{Q}_{2}$ ([15, Lemma 2.5]), and we deduce that the Witt index of $b_{4,1}$ is 1 over $\mathbb{Q}_{2}$. 
The rank of a spin group over a field of characteristic $\neq 2$ is the Witt index of the defining quadratic form. We deduce that

$$
\begin{aligned}
& \operatorname{rk}_{S} \underline{\operatorname{Spin}}\left(b_{4,1}\right)=\operatorname{rk}_{\mathbb{R}} \underline{\operatorname{Spin}}\left(b_{4,1}\right)+\mathrm{rk}_{\mathbb{Q}_{2}} \underline{\operatorname{Spin}}\left(b_{4,1}\right)=1+1=2, \\
& \operatorname{rk}_{S} \underline{\operatorname{Spin}}\left(b_{2,3}\right)=\operatorname{rk}_{\mathbb{R}} \underline{\operatorname{Spin}}\left(b_{2,3}\right)+\mathrm{rk}_{\mathbb{Q}_{2}} \underline{\operatorname{Spin}}\left(b_{2,3}\right)=2+2=4 .
\end{aligned}
$$

In particular, the $S$-arithmetic groups $\Delta_{1}=\operatorname{Spin}\left(b_{4,1}\right)(\mathbb{Z}[1 / 2])$ and $\Delta_{2}=\operatorname{Spin}\left(b_{2,3}\right)(\mathbb{Z}[1 / 2])$ have the congruence subgroup property (see also [32, Theorem 5]) and are hence profinitely isomorphic.

The symmetric space $X_{1}$ associated with $\operatorname{Spin}(4,1)$ has dimension 4 , whereas the symmetric space $X_{2}$ of $\operatorname{Spin}(2,3)$ has dimension 6. In particular, $\operatorname{dim} X_{1} \not \equiv \operatorname{dim} X_{2} \bmod 4$, and the useful Theorem 2 fails in the $S$-arithmetic case. However, the Euler characteristics of $\Delta_{1}$ and $\Delta_{2}$ nevertheless have the same sign. Using Serre's description of the Euler-Poincaré measure [56], we see that the sign of the Euler characteristic of $\Delta_{1}$ is

$$
\operatorname{sign}\left(\chi\left(\Delta_{1}\right)\right)=(-1)^{\operatorname{dim}\left(X_{1}\right) / 2} \cdot(-1)^{\mathrm{rk}_{\mathrm{Q}}\left(\underline{\left.\operatorname{Spin}\left(b_{4,1}\right)\right)}\right.}=-1
$$

and the sign of the Euler characteristic of $\Delta_{2}$ is

$$
\operatorname{sign}\left(\chi\left(\Delta_{2}\right)\right)=(-1)^{\operatorname{dim}\left(X_{2}\right) / 2} \cdot(-1)^{\mathrm{rk}_{\mathrm{Q}}\left(\underline{\operatorname{Spin}}\left(b_{2,3}\right)\right)}=-1
$$

The problem $\operatorname{dim} X_{1} \not \equiv \operatorname{dim} X_{2} \bmod 4$ is repaired by the change of the $\mathbb{Q}_{2}$-rank modulo 2: that is, $\mathrm{rk}_{\mathbb{Q}_{2}} \underline{\operatorname{Spin}}\left(b_{4,1}\right) \not \equiv \mathrm{rk}_{\mathbb{Q}_{2}} \underline{\operatorname{Spin}}\left(b_{2,3}\right) \bmod 2$.

Acknowledgements. Holger Kammeyer, Steffen Kionke, and Roman Sauer acknowledge funding by the Deutsche Forschungsgemeinschaft (DFG, German Research Foundation) - 281869850 (RTG 2229) and 338540207. Jean Raimbault acknowledges support from ANR grant ANR-16-CE40-0022-01 - AGIRA.

Conflict of Interest: None.

\section{References}

[1] I. Agol, 'The virtual Haken conjecture', Doc. Math. 18 (2013), 1045-1087, with an appendix by I. Agol, D. Groves, and J. Manning.

[2] M. Aka, 'Arithmetic groups with isomorphic finite quotients', J. Algebra 352 (2012), 322-340.

[3] M. Aka, 'Profinite completions and Kazhdan's property (T)', Groups Geom. Dyn. 6(2), 221-229, 2012.

[4] E. Artin, Geometric Algebra (Interscience Publishers, Inc., New York-London, 1957).

[5] G. Baumslag, 'Residually finite groups with the same finite images', Compositio Math. 29 (1974), $249-252$.

[6] N. Bergeron, F. Haglund, and D. T. Wise, 'Hyperplane sections in arithmetic hyperbolic manifolds', J. Lond. Math. Soc. 83(2) (2011), 431-448.

[7] M. Boileau and S. Friedl, 'The profinite completion of 3-manifold groups, fiberedness and the thurston norm' (2015).

[8] A. Borel, Introduction aux groupes arithmétiques (Publications de l'Institut de Mathématique de l'Université de Strasbourg, XV, Actualités Scientifiques et Industrielles, No. 1341, Hermann, Paris, 1969).

[9] A. Borel, 'The $L^{2}$-cohomology of negatively curved Riemannian symmetric spaces', Ann. Acad. Sci. Fenn. Ser. A I Math. 10 (1985), 95-105.

[10] A. Borel and J.-P. Serre, 'Corners and arithmetic groups', Comment. Math. Helv. 48 (1973), 436-491, avec un appendice: 'Arrondissement des variétés à coins', par A. Douady et L. Hérault.

[11] M. R. Bridson, M. D. E. Conder, and A. W. Reid, 'Determining Fuchsian groups by their finite quotients', Israel J. Math. 214(1) (2016), 1-41.

[12] M. R. Bridson, D. B. McReynolds, A. W. Reid, and R. Spitler, 'Absolute profinite rigidity and hyperbolic geometry', 2018.

[13] M. R. Bridson and A. W. Reid, 'Profinite rigidity, fibering, and the figure-eight knot', arXiv e-print, 2015.

[14] D. Burghelea, L. Friedlander, T. Kappeler, and P. McDonald, 'Analytic and Reidemeister torsion for representations in finite type Hilbert modules', Geom. Funct. Anal. 6(5) (1996), 751-859.

[15] J. W. S. Cassels, Rational quadratic forms, vol. 13 of London Mathematical Society Monographs (Academic Press, Inc. [Harcourt Brace Jovanovich, Publishers], London-New York, 1978).

[16] J. Cheeger and M. Gromov, ' $L_{2}$-cohomology and group cohomology', Topology 25(2) (1986), 189-215. 
[17] M. Demazure and P. Gabriel, 'Groupes algébriques, Tome I: Géométrie algébrique, généralités, groupes commutatifs, Masson and Cie, eds. (Paris; North-Holland Publishing Co., Amsterdam, 1970), avec un appendice it Corps de classes local par M. Hazewinkel.

[18] J. Dixon, M. du Sautoy, A. Mann, and D. Segal, Analytic pro-p Groups, Cambridge Studies in Advanced Mathematics, vol. 61 (Cambridge University Press, Cambridge, 2003).

[19] J. D. Dixon, E. W. Formanek, J. C. Poland, and L. Ribes, 'Profinite completions and isomorphic finite quotients', J. Pure Appl. Algebra 23(3) (1982) 227-231.

[20] H. M. Edwards, Riemann's Zeta Function, Pure and Applied Mathematics, vol. 58 (Academic Press, New York-London, 1974).

[21] A. V. Efremov, 'Cell decompositions and the Novikov-Shubin invariants', Uspekhi Mat. Nauk 46(3(279)) (1991), 189-190.

[22] A. Furman, 'A survey of measured group theory', in: Geometry, Rigidity, and Group Actions, Chicago Lectures in Math. (Univ. Chicago Press, Chicago, IL, 2011), 296-374.

[23] D. Gaboriau, 'Invariants $l^{2}$ de relations d'équivalence et de groupes', Publ. Math. Inst. Hautes Études Sci. (95) (2002), 93-150.

[24] P. Gille and P. Polo, editors, Schémas en groupes (SGA 3), Tome III Structure des schémas en groupes réductifs, Documents Mathématiques (Paris), vol. 8 (Société Mathématique de France, Paris, 2011), Séminaire de Géométrie Algébrique du Bois Marie, 1962-64.

[25] G. Harder, 'A Gauss-Bonnet formula for discrete arithmetically defined groups', Ann. Sci. École Norm. Sup. 4 (1971), 409-455.

[26] J. Hilgert and K.-H. Neeb, Structure and Geometry of Lie Groups, Springer Monographs in Mathematics (Springer, New York, 2012).

[27] J. E. Humphreys, Introduction to Lie algebras and Representation Theory, Graduate Texts in Mathematics, vol. 9 (SpringerVerlag, New York-Berlin, 1972).

[28] H. Kammeyer, ' $L^{2}$-invariants of nonuniform lattices in semisimple Lie groups', PhD thesis, Universität Göttingen (2013).

[29] H. Kammeyer, ' $L^{2}$-invariants of nonuniform lattices in semisimple Lie groups', Algebr. Geom. Topol. 14(4) (2014) 24752509.

[30] H. Kammeyer, 'Profinite commensurability of $S$-arithmetic groups', Acta Arith., to appear (2018).

[31] H. Kammeyer, Introduction to $\ell^{2}$-invariants, Lecture Notes in Mathematics, vol. 2247 (Springer, Cham, 2019).

[32] H. Kammeyer and R. Sauer, ' $S$-arithmetic spinor groups with the same finite quotients and distinct $\ell^{2}$-cohomology', Groups Geom. Dyn., to appear (2018).

[33] S. Kionke, 'Lefschetz numbers of involutions on arithmetic subgroups of inner forms of the special linear group', PhD thesis, Universität Wien (2012).

[34] S. Kionke, 'Lefschetz numbers of symplectic involutions on arithmetic groups', Pacific J. Math. 271(2) (2014), 369-414.

[35] S. Kionke, 'On lower bounds for cohomology growth in p-adic analytic towers', Math. Z. 277 (2014), 709-723.

[36] A. W. Knapp, Lie Groups Beyond an Introduction, 2nd ed, Progress in Mathematics, vol. 140 (Birkhäuser, Boston, 2002).

[37] M. Kneser, 'Normalteiler ganzzahliger Spingruppen', J. Reine Angew. Math. 311/312 (1979), 191-214.

[38] M.-A. Knus, Quadratic and Hermitian forms over rings, Grundlehren der Mathematischen Wissenschaften, vol. 294 (Springer-Verlag, Berlin, 1991), with a foreword by I. Bertuccioni.

[39] M.-A. Knus, A. Merkurjev, M. Rost, and J.-P. Tignol, The Book of Involutions, American Mathematical Society Colloquium Publications, vol. 44 (American Mathematical Society, Providence, RI, 1998), with a preface in French by J. Tits.

[40] R. E. Kottwitz, 'Tamagawa numbers', Ann. of Math. (2) 127(3) (1988) 629-646.

[41] A. Lubotzky and D. Segal, Subgroup Growth, Progress in Mathematics, vol. 22 (Birkhäuser Verlag, Basel, 2003).

[42] W. Lück, $L^{2}$-Invariants: Theory and Applications to Geometry and K-Theory, Results in Mathematics and Related Areas (3) (Springer-Verlag, Berlin, 2002).

[43] W. Lück, R. Sauer, and C. Wegner, ' $L^{2}$-torsion, the measure-theoretic determinant conjecture, and uniform measure equivalence', J. Topol. Anal. 2(2) (2010), 145-171.

[44] G. A. Margulis, Discrete Subgroups of Semisimple Lie Groups, Results in Mathematics and Related Areas (3), vol. 17 (Springer-Verlag, Berlin, 1991).

[45] J. S. Milne, Algebraic Groups, Cambridge Studies in Advanced Mathematics, vol. 170 (Cambridge University Press, Cambridge, 2017).

[46] A. Minasyan and P. Zalesskii, 'Virtually compact special hyperbolic groups are conjugacy separable', Comment. Math. Helv. 91(4) (2016) 609-627.

[47] J. Neukirch, Algebraic Number Theory, Grundlehren der Mathematischen Wissenschaften, vol. 322 (Springer-Verlag, Berlin, 1999).

[48] M. Olbrich, ' $L^{2}$-invariants of locally symmetric spaces', Doc. Math. 7 (2002), 219-237.

[49] V. Platonov and A. Rapinchuk, Algebraic Groups and Number Theory, Pure and Applied Mathematics, vol. 139 (Academic Press, Inc., Boston, 1994).

[50] M. S. Raghunathan, 'The congruence subgroup problem', Proc. Indian Acad. Sci. Math. Sci. 114(4), $299-308,2004$.

[51] A. Rapinchuk, 'The congruence subgroup problem for algebraic groups', in: Topics in Algebra, Part 2 (Warsaw, 1988), vol. 26 (PWN, Warsaw, 1990), 399-410. 
[52] A. S. Rapinchuk, Y. Segev, and G. M. Seitz, 'Finite quotients of the multiplicative group of a finite dimensional division algebra are solvable', J. Amer. Math. Soc. 15(4) (2002), 929-978.

[53] L. Ribes and P. Zalesskii, Profinite Groups, Results in Mathematics and Related Areas (3), vol. 40, 2nd ed . (Springer-Verlag, Berlin, 2010).

[54] J. Rohlfs and B. Speh, 'Automorphic representations and Lefschetz numbers', Ann. Sci. Éc. Norm. Supér. 22(3) (1989), 473-499.

[55] W. Scharlau, Quadratic and Hermitian Forms, Grundlehren der math. Wiss. 270 (Springer-Verlag, Berlin, 1985).

[56] J.-P. Serre, 'Cohomologie des groupes discrets', Ann. of Math. Studies 70 (1971), 77-169.

[57] J.-P. Serre, 'Groupes de congruence' (d'après H. Bass, H. Matsumoto, J , Mennicke, J. Milnor, C. Moore) in: Séminaire Bourbaki, vol. 10 (Soc. Math. France, Paris, 1995), Exp. No. 330, 275-291.

[58] M. Stover, 'Lattices in PU $(n, 1)$ that are not profinitely rigid', Proc. Amer. Math. Soc. 147(12) (2019), 5055-5062. 